\title{
Significant Wave Height Prediction with the CRBM-DBN Model
}

\author{
XINING ZHANG \\ Fujian Key Laboratory of Light Propagation and Transformation, College of Information Science and \\ Engineering, Huaqiao University, Xiamen, China \\ HAO DAI \\ Institute of Ocean Exploration Technology, College of Ocean and Earth Sciences, Xiamen University, \\ Xiamen, China
}

(Manuscript received 10 August 2018, in final form 30 November 2018)

\begin{abstract}
In recent years, deep learning technology has been gradually used for time series data prediction in various fields. In this paper, the restricted Boltzmann machine (RBM) in the classical deep belief network (DBN) is substituted with the conditional restricted Boltzmann machine (CRBM) containing temporal information, and the CRBM-DBN model is constructed. Key model parameters, which are determined by the particle swarm optimization (PSO) algorithm, are used to predict the significant wave height. Observed data in 2016, which are from nearshore and offshore buoys (i.e., 42020 and 42001) belonging to the National Data Buoy Center (NDBC), are taken to train the model, and the corresponding data in 2017 are used for testing with lead times of 1-24 h. In addition, we trained the data of 42040 in 2003 and tested the data in 2004 in order to investigate the prediction ability of the CRBM-DBN model for the extreme event. The prediction ability of the model is evaluated by the Nash-Sutcliffe coefficient of efficiency (CE) and root-mean-square error (RMSE). Experiments demonstrate that for the short-term $(\leq 9 \mathrm{~h})$ prediction, the RMSE and CE for the significant wave height prediction are $<10 \mathrm{~cm}$ and $>0.98$, respectively. Moreover, the relative error of the short-term prediction for the maximum wave height is less than $26 \%$. The excellent short-term and extreme events forecasting ability of the CRBM-DBN model is vital to ocean engineering applications, especially for designs of ocean structures and vessels.
\end{abstract}

\section{Introduction}

The accurate prediction of ocean wave parameters, for example, wave height and wave period, is of vital importance for project, design, use, and maintenance of structures in offshore and coastal engineering. Wave height observation, which varies with time, is a kind of typical time series data. The prediction techniques based on mathematical models and data are mainly applied to the wave height prediction.

In general, the wave prediction based on mathematical models is realized by wave numerical models, such as the Wave Model (WAM; WAMDI Group 1988), Simulating Waves Nearshore (SWAN; Booij et al. 1999; Ris et al. 1999), and WAVEWATCH-III (WW3; Tolman 1989). However, numerical models are often approximate or insufficient, and accurate enough water depth measurements

Corresponding author: Hao Dai, daihaozxn@163.com. are required in advance. Therefore, the complicated and changeable wave data are difficult to deal with by the method in actual applications. The prediction precision is limited and the computational cost is very high.

The data-driven prediction methods for the time series data, which are independent of mathematical and physical models of study objects, examine inherent laws of data characteristics and forecast. The mature techniques are mainly based on supporting vector machine (SVM; Mahjoobi and Mosabbe 2009), filters (Altunkaynak and Özger 2004), genetic algorithm (GA; Gaur and Deo 2008; Cañellas et al. 2010), fuzzy logic (Özger and Sen 2007), and the hybrid of wavelet and fuzzy logic (Özger 2010).

In recent years oceanographers have frequently attempted to design various neural networks or hybrid models to predict wave height. With wind data as the input, Deo et al. (2001) investigated the predictive ability of neural nets to wave height data, while with wave data as the input, Tsai et al. (2002), Makarynskyy (2004), 


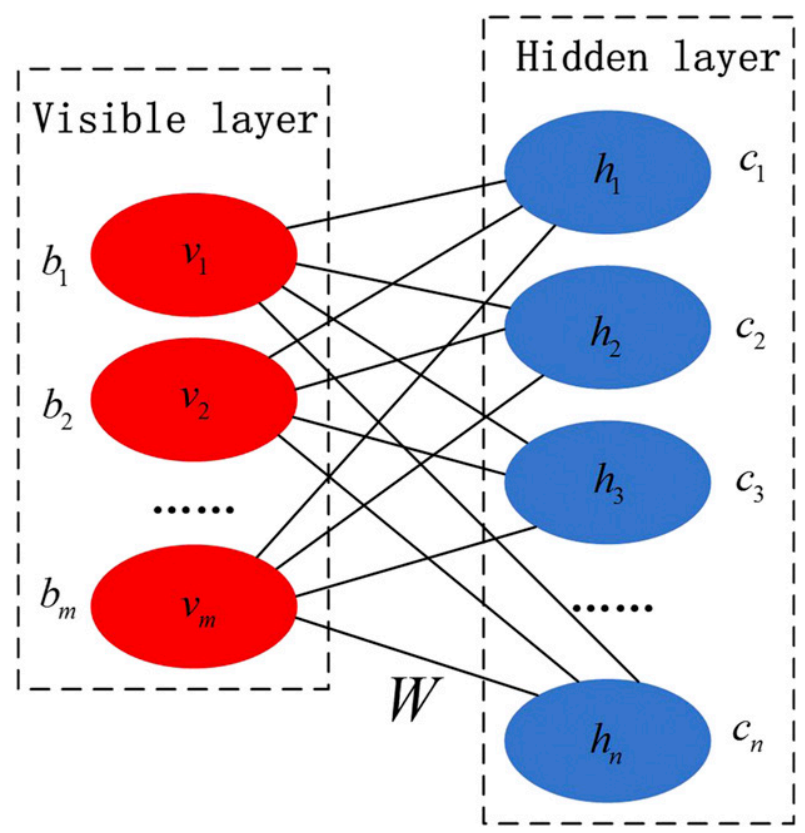

FIG. 1. Standard structure of RBM, where $v_{1}, v_{2}, \ldots, v_{m}$ and $h_{1}, h_{2}, h_{3}, \ldots, h_{n}$ represent neurons in the visible and latent layer, respectively; $b_{1}, b_{2}, \ldots, b_{m}$ and $c_{1}, c_{2}, c_{3}, \ldots, c_{n}$ are bias for the visible and latent layer, respectively; and $\mathbf{W}$ represents the weight matrix between the visible and hidden layers.

and Londhe and Panchang (2006) designed neural networks to forecast wave height. Mandal and Prabaharan (2006) predicted the wave height by temporal univariate modeling and the recurrent neural network. According to the meteorological data, Günaydin (2008) predicted monthly mean significant wave heights by using neural network and regression methods. Malekmohamadi et al. (2008), Londhe et al. (2016), and Deshmukh et al. (2016) combined neural network and numerical models to realize the wave height prediction. To solve the time lag and the lack of extreme wave height prediction ability in the neural network, Deka and Prahlada (2012), Dixit and Londhe (2016), and Dixit et al. (2015) added the wavelet into the neural network.

From other papers on wave height prediction by machine learning methods, for example, a neural network, it is easy to find that the models used in these methods generally have shallow architecture, that is, the hidden layer number is 1 . In addition, the hidden layer has fewer neurons. To approach sufficiently arbitrary multivariate nonlinear functions, the neural network should have deep enough levels or sufficient neural units. In fact, however, with the number of hidden layers increasing, when random values are used as initial weights, problems will arise. Weights will fall into a local minimum (not global minimum) in the training process if

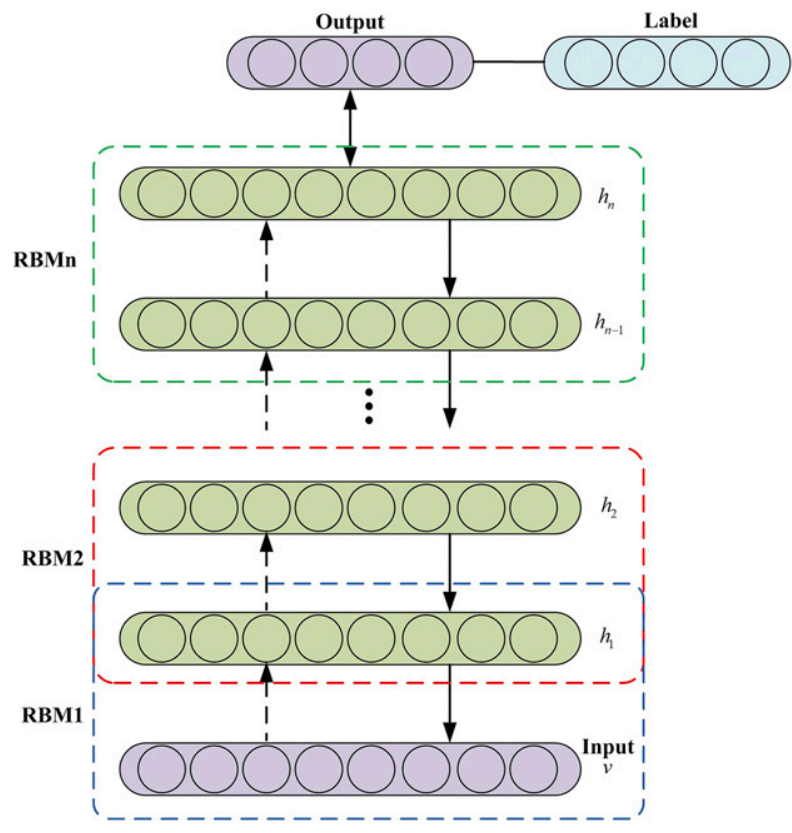

FIG. 2. Structure of DBN.

initial weights are oversized, while if initial weights are undersized, gradient values are tiny when errors propagate to the front layers, and they are unable to reach optimal values as the changes of weights are tiny (Hinton and Salakhutdinov 2006). Moreover, if the shallow network has excessive neural units, then accordingly, each neural unit requires many training samples to adjust (Bengio 2009). In practice, therefore, the biggest limitation of machine learning methods with the shallow architecture lies in the finite representative ability for complicated functions with finite samples and neural units. In other words, for sophisticated problems, the shallow machine learning has disadvantages of weak abilities for feature extraction, functions expression, and generalization.

For making up for the various deficiencies in the shallow machine learning, Hinton et al. (2006) introduced the "deep learning" concept and proposed a fast-learning algorithm for deep belief networks (DBNs). A DBN is a generative model for solving the difficult inference of logistic belief nets with many latent layers. Complicated functions can be expressed with fewer parameters since DBN has multiple hidden layers. In the meanwhile, the layer-wise initialization is used to solve the training problem of neural networks with many latent layers. Hence, DBN has the advantages of excellent feature learning and expression for complicated functions.

The traditional DBN consists of many binary restricted Boltzmann machines (RBMs) and is suitable for 


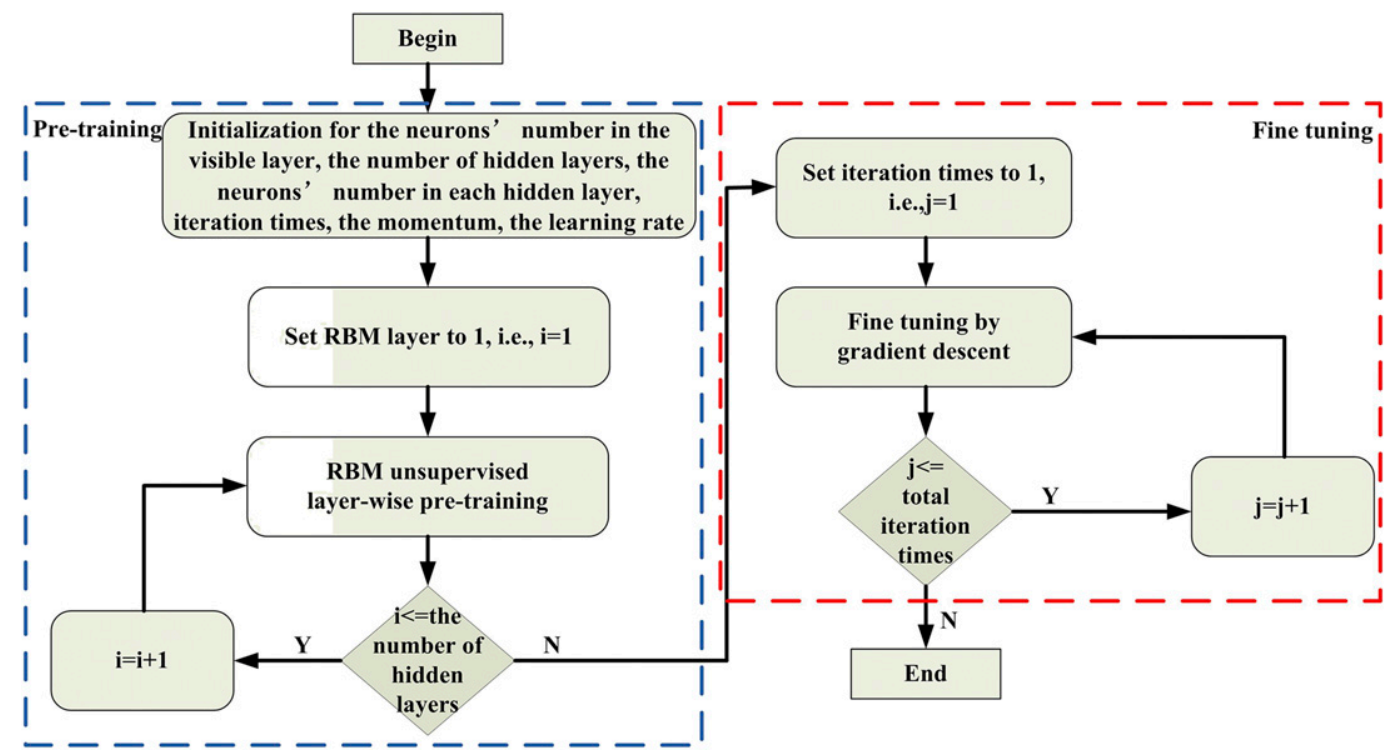

FIG. 3. Training process of DBN.

modeling static data (Längkvist et al. 2014). At present researchers combine the DBN with other techniques (e.g., SVM and GA) to predict one-dimensional time series data. Research domains involve nature (e.g., flow, red tide; Chen et al. 2012; Bai et al. 2016), economics (e.g., stocks, exchange rate; Shen et al. 2015), energy (e.g., energy consumption; Qiu et al. 2017; Mocanu et al. 2016), and society (e.g., traffic flow; Koesdwiady et al. 2016; Huang et al. 2014).

Because of the conditional restricted Boltzmann machine (CRBM) considering the temporal information between data in/between layers, we substitute CRBM for RBM and combine this with DBN to construct the CRBM-DBN model in this paper. The particle swarm optimization (PSO) algorithm is employed to determine key model parameters, that is, the number of neurons in the input layer, the number of hidden layers, and the number of neurons in every hidden layer. By virtue of significant wave height data measured by near- and offshore wave buoys of National Data Buoy Center (NDBC) in the Gulf of Mexico, the models are trained by the pattern "wave-wave" and the accurate short-term predictions for the wave height are achieved. (Unless stated specifically, "wave height" hereinafter means "significant wave height.")

\section{Methodology}

\section{a. $D B N$}

The classical DBN is composed of multi-RBM stacks and a classification or regression layer. As a discriminative model, the application of DBN to the prediction of wave height time series data is mainly discussed in the paper. In this context, the DBN realizes the model training by the fast and greedy forward-learning and backward-propagation fine-tuning mechanism with gradient descent. The structure and training process of the RBM that is the kernel of DBN is introduced at first.

\section{1) $\mathrm{RBM}$}

RBM, which developed from the Boltzmann machine, canceled connections between layers and retained ones in layers for simplifying models and convenient inference. A standard RBM structure consists of a visible layer and a hidden one. As shown in Fig. 1, neurons in layers are binary.

By virtue of the activation function, the RBM converts the input vector, which is from the visible or previous hidden layer, to the hidden layer; minimizes the internal energy function by training; and completes feature extraction. The data vector obtained by the hidden layer is considered to be another best expression for the original input one.

RBM is a model based on energy. According to Fig. 1, suppose $\mathbf{v}=\left\{v_{1}, v_{2}, \ldots, v_{m}\right\}, \mathbf{h}=\left\{h_{1}, h_{2}, h_{3}, \ldots, h_{n}\right\}$, $\mathbf{b}=\left\{b_{1}, b_{2}, \ldots, b_{m}\right\}, \mathbf{c}=\left\{c_{1}, c_{2}, c_{3}, \ldots, c_{n}\right\}$, the model parameter $\theta=\{\mathbf{W}, \mathbf{b}, \mathbf{c}\}$, and the energy function of the status $\{\mathbf{v}, \mathbf{h}\}$ is (Salakhutdinov 2009)

$$
\begin{aligned}
E(\mathbf{v}, \mathbf{h} ; \theta) & =-\mathbf{v}^{\mathrm{T}} \mathbf{W h}-\mathbf{b}^{\mathrm{T}} \mathbf{v}-\mathbf{c}^{\mathrm{T}} \mathbf{h} \\
& =-\sum_{i} \sum_{j} W_{i j} v_{i} h_{j}-\sum_{i} b_{i} v_{i}-\sum_{j} c_{j} h_{j} ;
\end{aligned}
$$


here $v_{i}, h_{j}$ are the neurons $i$ and $j$ in the visible and hidden layers; $b_{i}, c_{j}$ are the biases of $v_{i}$ and $h_{j}$; and $W_{i j}$ is the weight coefficient between $v_{i}$ and $h_{j}$.

The joint distribution over the visible and hidden units is defined by

$$
P(\mathbf{v}, \mathbf{h} ; \theta)=\frac{1}{Z(\theta)} \exp [-E(\mathbf{v}, \mathbf{h} ; \theta)],
$$

where the partition function or normalizing constant is $Z(\theta)=\sum_{\mathbf{v}} \sum_{\mathbf{h}} \exp [-E(\mathbf{v}, \mathbf{h} ; \theta)]$.

For an actual problem, our most concern is about the probability distribution of the observation data $\mathbf{v}$. It corresponds to the marginal distribution of $P(\mathbf{v}, \mathbf{h} ; \theta)$ and is also known as the likelihood function. In detail, Eq. (3) is confirmed:

$$
\begin{aligned}
P(\mathbf{v} ; \theta) & =\sum_{\mathbf{h}} P(\mathbf{v}, \mathbf{h} ; \theta) \\
& =\frac{1}{Z(\theta)} \sum_{\mathbf{h}} \exp [-E(\mathbf{v}, \mathbf{h} ; \theta)] .
\end{aligned}
$$

Similarly,

$$
\begin{aligned}
P(\mathbf{h} ; \theta) & =\sum_{\mathbf{v}} P(\mathbf{v}, \mathbf{h} ; \theta) \\
& =\frac{1}{Z(\theta)} \sum_{\mathbf{v}} \exp [-E(\mathbf{v}, \mathbf{h} ; \theta)] .
\end{aligned}
$$

It is required to compute $Z(\theta)$, and the calculated amount is huge for determining $P(\mathbf{v} ; \theta)$ and $P(\mathbf{h} ; \theta)$. Even if the model parameter $\theta$ can be obtained by training; therefore, the distribution determined by $\theta$ is unable to be computed effectively. Connections between the layer of hidden and visible variables exist in RBM while ones between two variables of the same layer do not exist. It means that when the statuses of neural units in the visible layer are given, the activation conditions of neural units in each hidden layer are independent and vice versa. Hence (Salakhutdinov 2009),

$$
P(\mathbf{h} \mid \mathbf{v} ; \theta)=\prod_{j} p\left(h_{j} \mid \mathbf{v}\right),
$$

and

$$
P(\mathbf{v} \mid \mathbf{h} ; \theta)=\prod_{i} p\left(v_{i} \mid \mathbf{h}\right)
$$

When the statuses of the visible neurons are given, the activation probability of the $j$ th neuron in the hidden layer is

$$
p\left(h_{j}=1 \mid \mathbf{v}\right)=\sigma\left(\sum_{i} W_{i j} v_{i}+c_{j}\right)
$$

here the sigmoid activation function is $\sigma(x)=$ $1 /[1+\exp (-x)]$.

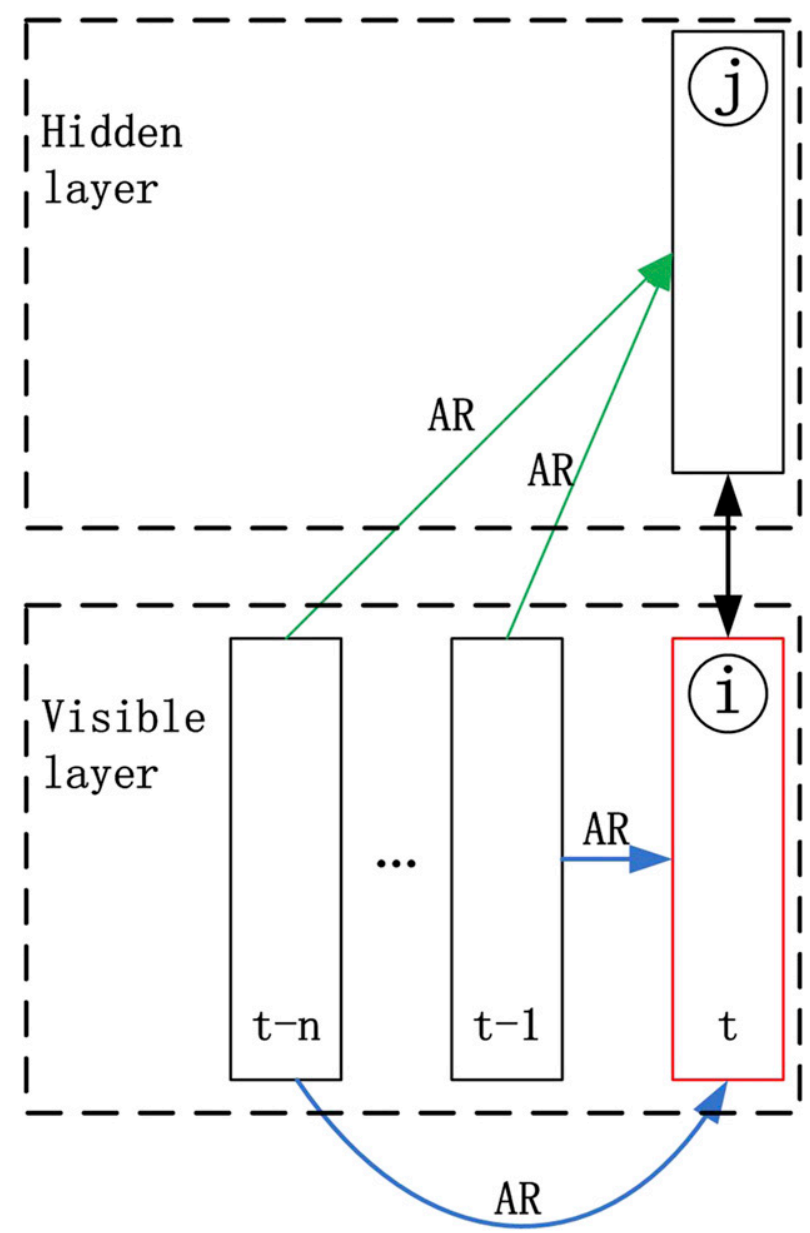

FIG. 4. Structure of CRBM.

In the same way, when the statuses of the hidden units are given, the activation probability of the $i$ th neuron in the visible layer is

$$
p\left(v_{i}=1 \mid \mathbf{h}\right)=\sigma\left(\sum_{j} W_{i j} h_{j}+b_{i}\right) .
$$

The purpose of the RBM training is to determine the optimal model parameter $\theta$ by maximizing the likelihood function, that is, Eq. (9), with the gradient ascent method. Given that training samples are fitted so that the probability distribution represented by $\theta$ coincides with training data as much as possible (Fischer and Igel 2012):

$$
\ln L(\theta)=\ln \prod_{i=1}^{m} p\left(v_{i}\right)=\sum_{i=1}^{m} \ln p\left(v_{i}\right)
$$

Instead of the traditional Gibbs sampling, Hinton (2002) proposed a fast algorithm named contrastive 


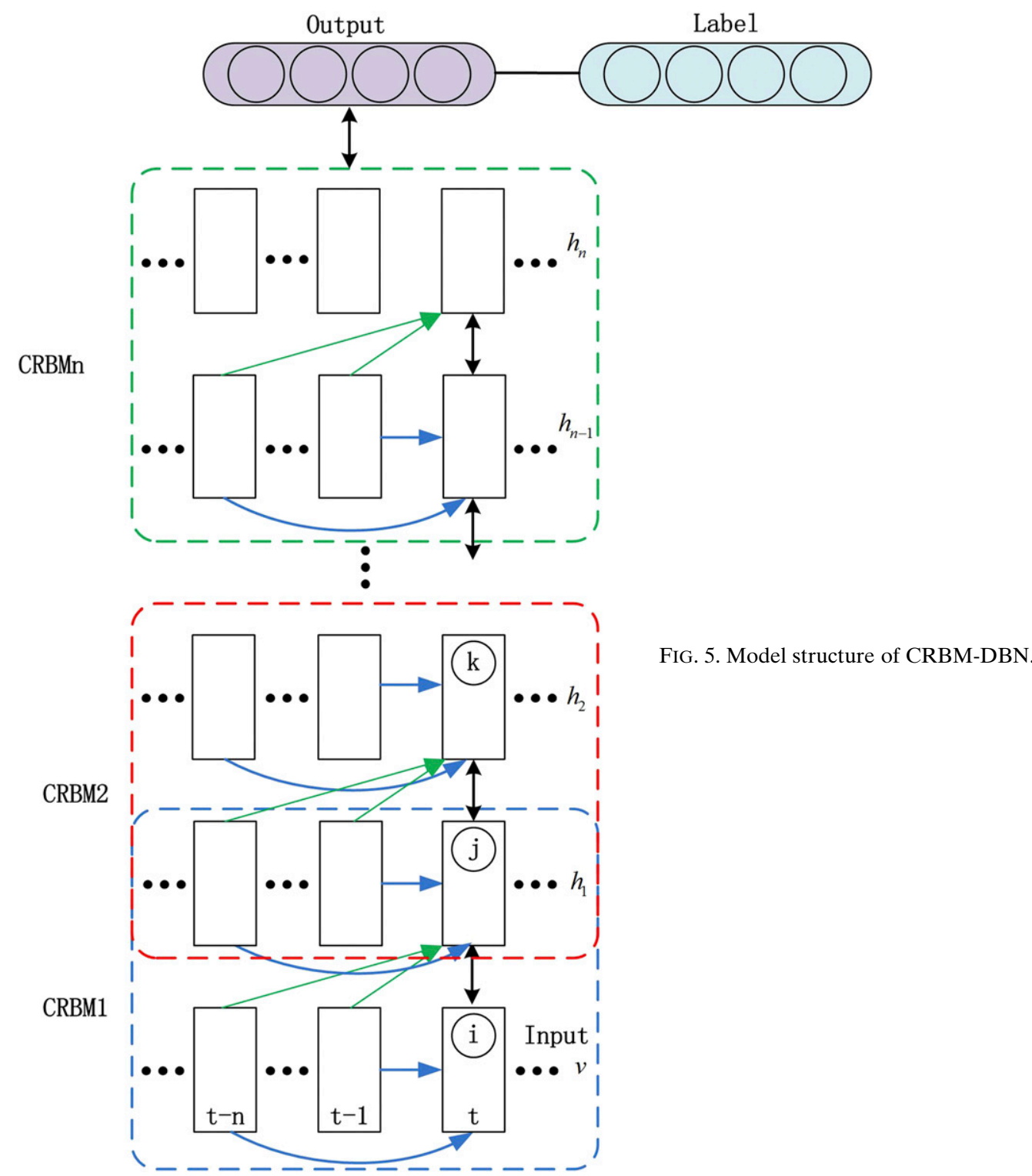

divergence $(\mathrm{CD})$ to learn and update the model parameter $\theta$. The key processes of the $\mathrm{CD}$ algorithm are 1 ) the features of the training samples are projected from the visible layer to the hidden one by Eq. (7), 2) the output of the hidden layer is reconstructed to the visible one based on Eq. (8) and the error between the input and reconstruction data is computed, and 3) model parameters are updated by maximizing the logarithm

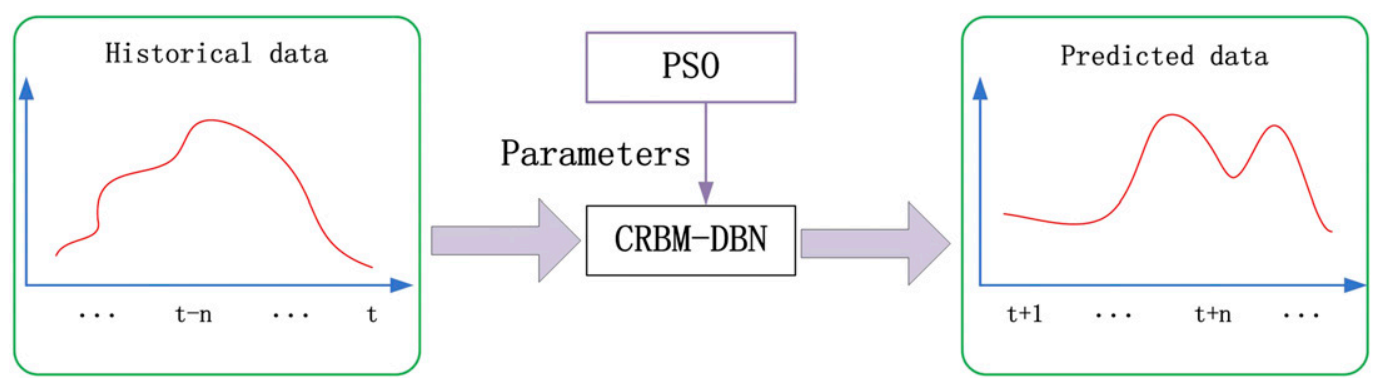

FIG. 6. Schematic illustration for the wave height prediction. 


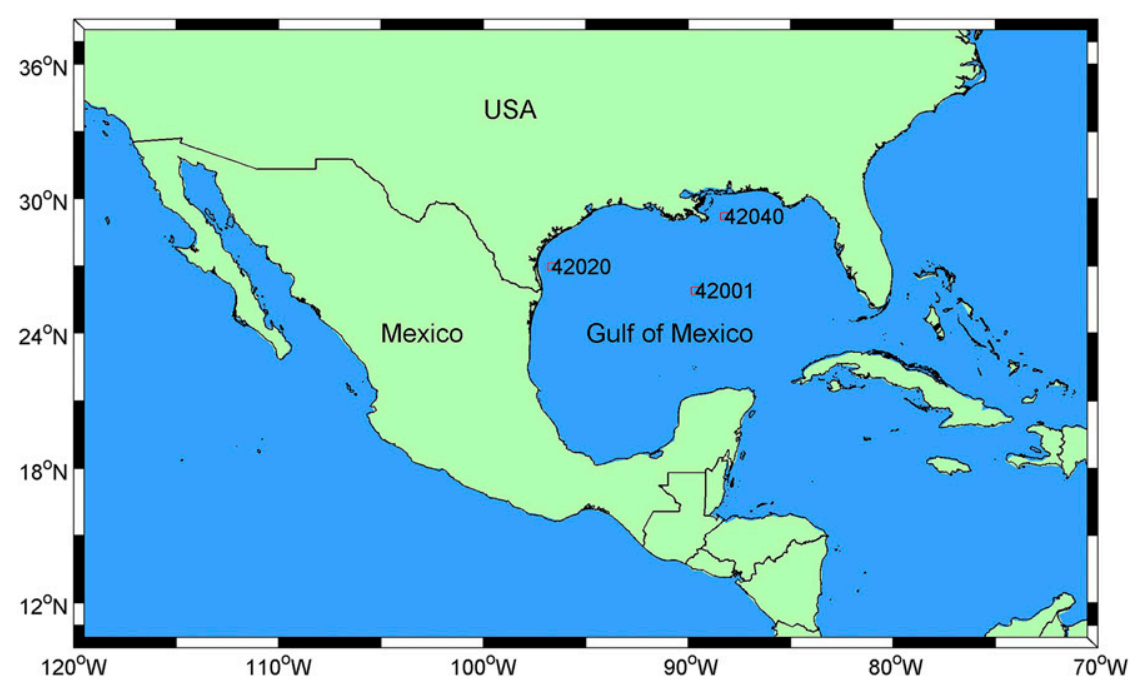

FIG. 7. Study area

likelihood function with random gradient ascent and the feature extraction is achieved. With the learning rate $\varepsilon$ and momentum $M$ considered (Hinton 2010), the main procedure of the $\mathrm{CD}$ algorithm for updating RBM is as follows:

1) It is supposed that the initial status of the visible units is $v_{1}=x_{0}$ and the initial values of $\mathbf{W}, \mathbf{b}, \mathbf{c}, \Delta \mathbf{W}, \Delta \mathbf{b}$, and $\Delta \mathbf{c}$ are all zero, where $\mathbf{W}$ is the connection weight between the visible and hidden layer and $\mathbf{b}$ and $\mathbf{c}$ represent the biases for the visible and hidden layer while $\Delta \mathbf{W}, \Delta \mathbf{b}$, and $\Delta \mathbf{c}$ represent corresponding updates for $\mathbf{W}, \mathbf{b}$, and $\mathbf{c}$, respectively.

2) For all hidden units $j=1,2, \ldots, n$, compute $P\left(h_{1 j}=\right.$ $\left.1 \mid v_{1}\right)$, and sample $h_{1 j}$ from $P\left(h_{1 j}=1 \mid v_{1}\right)$.

3) For all visible units $i=1,2, \ldots, m$, compute $P\left(v_{2 i}=\right.$ $\left.1 \mid h_{1}\right)$, and sample $v_{2 i}$ from $P\left(v_{2 i}=1 \mid h_{1}\right)$.

4) For all hidden units $j=1,2, \ldots, n$, compute $P\left(h_{2 j}=\right.$ $\left.1 \mid v_{2}\right)$.

5) Update all parameters according to Eq. (10):

$$
\begin{gathered}
\Delta \mathbf{W} \leftarrow M \Delta \mathbf{W}+\varepsilon\left[P\left(h_{1 j}=1 \mid v_{1}\right) v_{1}-P\left(h_{2 j}=1 \mid v_{2}\right) P\left(v_{2 i}=1 \mid h_{1}\right)\right], \\
\Delta \mathbf{b} \leftarrow M \Delta \mathbf{b}+\varepsilon\left[v_{1}-P\left(v_{2 i}=1 \mid h_{1}\right)\right], \\
\Delta \mathbf{c} \leftarrow M \Delta \mathbf{c}+\varepsilon\left[P\left(h_{1 j}=1 \mid v_{1}\right)-P\left(h_{2 j}=1 \mid v_{2}\right)\right], \\
\mathbf{W} \leftarrow \mathbf{W}+\Delta \mathbf{W}, \\
\mathbf{b} \leftarrow \mathbf{b}+\Delta \mathbf{b}, \\
\mathbf{c} \leftarrow \mathbf{c}+\Delta \mathbf{c} .
\end{gathered}
$$

\section{2) $\mathrm{DBN}$}

\begin{tabular}{|c|c|c|c|c|c|c|c|c|c|c|}
\hline \multirow[b]{2}{*}{ ID } & \multirow[b]{2}{*}{ Longitude } & \multirow[b]{2}{*}{ Latitude } & \multirow[b]{2}{*}{$\begin{array}{c}\text { Water } \\
\text { depth }(m)\end{array}$} & \multicolumn{7}{|c|}{ Wave height data characteristics } \\
\hline & & & & Year & $\begin{array}{l}\text { Total data } \\
\text { number }\end{array}$ & $\begin{array}{l}\text { Sampling output } \\
\text { period }(\mathrm{h})\end{array}$ & $\operatorname{Maximum}(\mathrm{m})$ & Minimum (m) & Mean (m) & $\begin{array}{l}\text { Standard } \\
\text { error }(\mathrm{m})\end{array}$ \\
\hline \multirow[t]{2}{*}{42001} & $89.668^{\circ} \mathrm{W}$ & $25.897^{\circ} \mathrm{N}$ & 3207 & 2016 & 6591 & 1 & 3.75 & 0.25 & 1.014 & 0.554 \\
\hline & & & & 2017 & 8095 & 1 & 5.92 & 0.15 & 1.155 & 0.736 \\
\hline \multirow[t]{2}{*}{42020} & $96.694^{\circ} \mathrm{W}$ & $26.969^{\circ} \mathrm{N}$ & 81 & 2016 & 8705 & 1 & 5.07 & 0.17 & 1.193 & 0.592 \\
\hline & & & & 2017 & 6792 & 1 & 7.34 & 0.18 & 1.302 & 0.672 \\
\hline \multirow[t]{2}{*}{42040} & $88.226^{\circ} \mathrm{W}$ & $29.208^{\circ} \mathrm{N}$ & 183 & 2003 & 8669 & 1 & 4.42 & 0.15 & 0.998 & 0.632 \\
\hline & & & & 2004 & 7517 & 1 & 15.96 & 0.15 & 1.087 & 0.836 \\
\hline
\end{tabular}

6) Repeat the training steps $2-5$ for $T$ periods and output the updated $\mathbf{W}, \mathbf{b}$, and $\mathbf{c}$ to the end.
The explaining-away effects are eliminated by "complementary priors" in DBN, and the inference of the

TABLE 1. Exact locations, water depths, and data characteristics for 42001, 42020, and 42040. 
TABLE 2. CRBM-DBN model parameters for 42001, 42020, and 42040. Bold indicates the best structure parameters by PSO searching.

\begin{tabular}{|c|c|c|c|c|}
\hline \multicolumn{2}{|c|}{ Buoy ID } & \multirow{2}{*}{$\frac{42001}{4}$} & \multirow{2}{*}{$\frac{42020}{3}$} & \multirow{2}{*}{$\frac{42040}{4}$} \\
\hline CRBM-DBN model parameters & No. of neurons in the input layer & & & \\
\hline & No. of hidden layers & 2 & 2 & 2 \\
\hline & No. of neurons in the first hidden layer & 6 & 8 & 7 \\
\hline & No. of neurons in the second hidden layer & 4 & 5 & 5 \\
\hline & No. of neurons in the output layer & 1 & 1 & 1 \\
\hline & Momentum in the pretraining & 0.9 & 0.9 & 0.9 \\
\hline & Learning rate in the pretraining & 0.01 & 0.01 & 0.01 \\
\hline
\end{tabular}

multilayer logistic network is achieved. As shown in Fig. 2, from the visible layer $\mathbf{v}$ to the secondary high layer $h_{n}$, an RBM is composed of every two adjacent layers. With the hidden layer output of the lower RBM as the visible layer input of the higher RBM, the unsupervised layer-wise pretraining is performed by stacking RBMs, and the feature extraction of the input data is obtained. Finally, the feature extracted by RBMs is mapped to the label through the activation function and the accurate prediction is realized by the supervised fine tuning (Hinton et al. 2006).

The complete training process of DBN contains two stages: pretraining and fine tuning. Its flowchart is illustrated as Fig. 3.

The unsupervised greedy pretraining algorithm proposed by Hinton et al. (2006) limits the training to the two adjacent layers. The deep network is divided into several shallow ones in this way, and the pretraining for the entire network is achieved by training these RBMs. In the fine-tuning stage, the model parameters obtained by the pretraining are used as the initial values in the DBN. From bottom to top, the final output is achieved by layer-wise mapping. The whole DBN network is finetuned by the error, which is obtained by comparing the final output with the label information and propagating to every RBM from top to bottom. The fine-tuning process of the DBN is similar to the one of the backpropagation (BP) neural network, and the only difference is the initialization with parameters obtained by the layer-wise training for the DBN, which overcomes some disadvantages such as falling into local minimum easily and overlong training time from random initialization parameters of the BP neural network.

\section{b. $C R B M$}

Typically, the RBM uses binary logistic units for visible and latent variables. For the prediction problem, however, Gaussian RBM should be introduced because the binarization is adverse to the continuous data operation. In other words, the visible variables satisfy the Gaussian distribution while the hidden ones still keep binary Bernoulli distribution. So the energy function of the status $\{\mathbf{v}, \mathbf{h}\}$ becomes (Salakhutdinov 2009)

$E(\mathbf{v}, \mathbf{h} ; \theta)=\sum_{i} \frac{\left(v_{i}-b_{i}\right)^{2}}{2 \sigma_{i}^{2}}-\sum_{i} \sum_{j} W_{i j} h_{j} \frac{v_{i}}{\sigma_{i}}-\sum_{j} c_{j} h_{j}$,

where $\theta=\{\mathbf{W}, \mathbf{b}, \mathbf{c}, \boldsymbol{\sigma}\}$ and $\sigma_{i}$ represent the standard error of Gaussian distribution for the visible unit $v_{i}$. In practice, $\sigma_{i}^{2}=1$ is generally for better results (a)

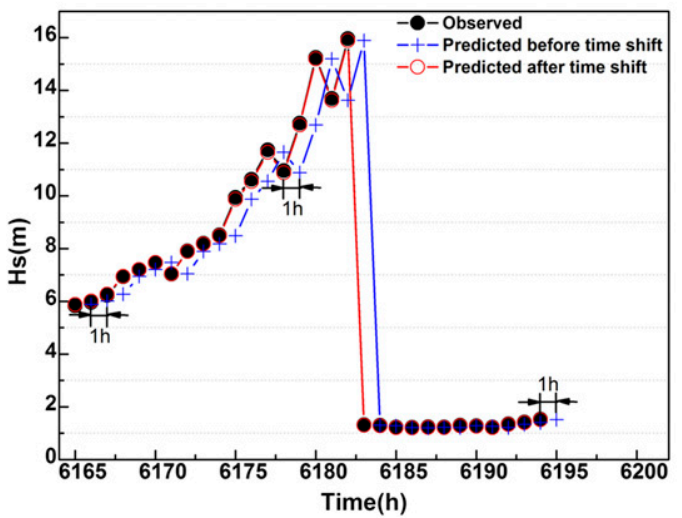

(b)

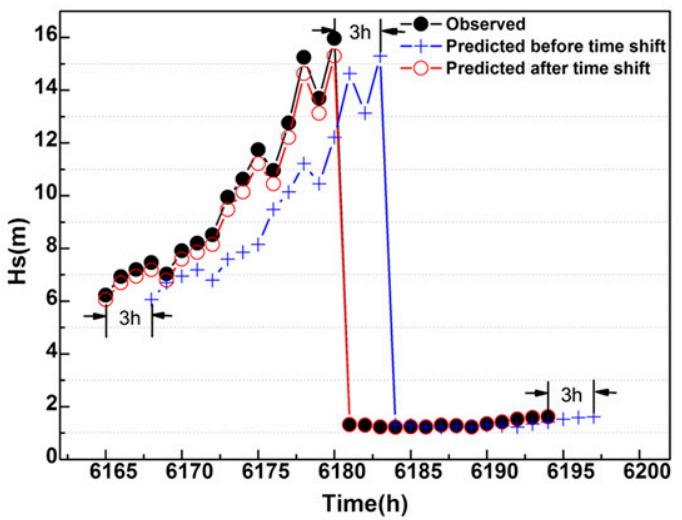

FIG. 8. Comparisons among the predicted before and after time shift and the observed time series for 42040: lead time (a) 1 and (b) $3 \mathrm{~h}$. 
(a)

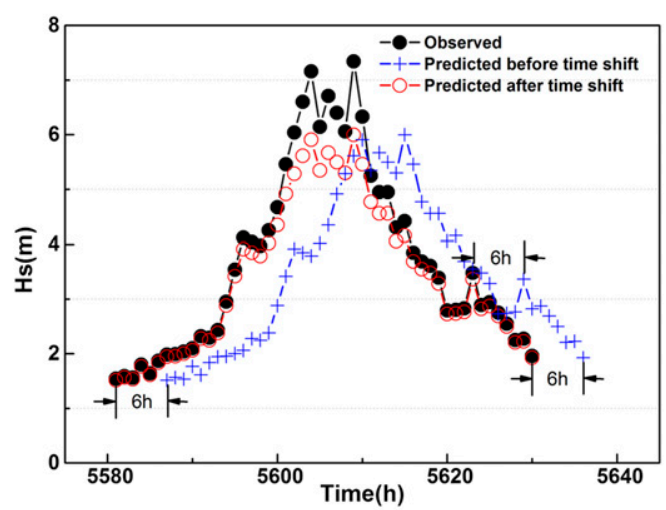

(b)

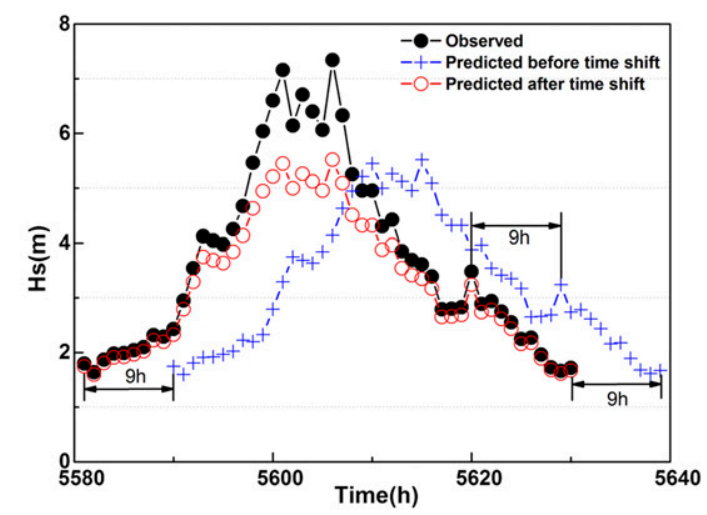

FIG. 9. Comparisons among the predicted before and after time shift and the observed time series for 42020: lead time (a) 6 and (b) $9 \mathrm{~h}$.

(Taylor et al. 2006). Hence, the activation probabilities of the updated $h_{j}$ and $v_{i}$ are

$$
p\left(h_{j}=1 \mid \mathbf{v}\right)=\sigma\left(\sum_{i} W_{i j} v_{i}+c_{j}\right)
$$

and

$$
\begin{aligned}
p\left(v_{i}=x \mid \mathbf{h}\right) & =N\left(b_{i}+\sigma_{i} \sum_{j} h_{j} W_{i j}, \sigma_{i}^{2}\right) \\
& =N\left(b_{i}+\sum_{j} h_{j} W_{i j}, 1\right) \\
& =\frac{1}{\sqrt{2 \pi}} \exp \left[-\frac{\left(x-b_{i}-\sum_{j} h_{j} W_{i j}\right)^{2}}{2}\right],
\end{aligned}
$$

\section{(a)}

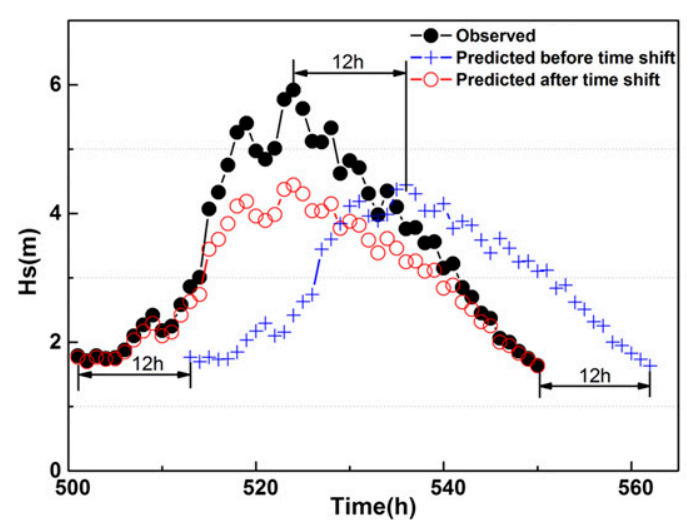

where $N(\mu, V)$ represents the Gaussian function with the mean $\mu$ and variance $V$.

As shown in Fig. 4, the temporal information has been added. Directed autoregressive (AR) connections are established from the visible units at historical time $t-n$ to the visible and hidden ones at current time $t$. These additional connections turn RBM into CRBM (Taylor et al. 2006).

The visible units at time $t-n$ make supplements, which dynamically update biases, to the visible and hidden ones at time $t$. Equations for updating biases dynamically are as follows:

$$
b \_\operatorname{star}_{i}=b_{i}+\sum_{k} \sum_{q} \alpha_{k i}^{t-q} v_{k}^{t-q}
$$

and

$$
c \_\operatorname{star}_{j}=c_{j}+\sum_{k} \sum_{q} \beta_{k j}^{t-q} v_{k}^{t-q},
$$

(b)

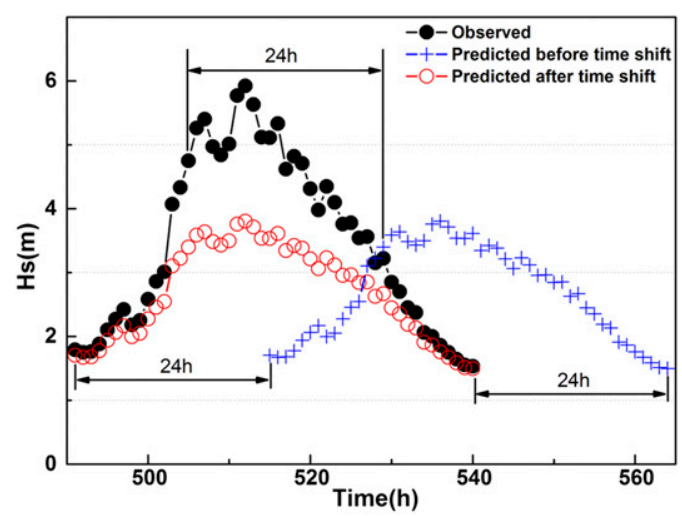

FIG. 10. Comparisons among the predicted before and after time shift and the observed time series for 42001: lead time (a) 12 and (b) $24 \mathrm{~h}$. 

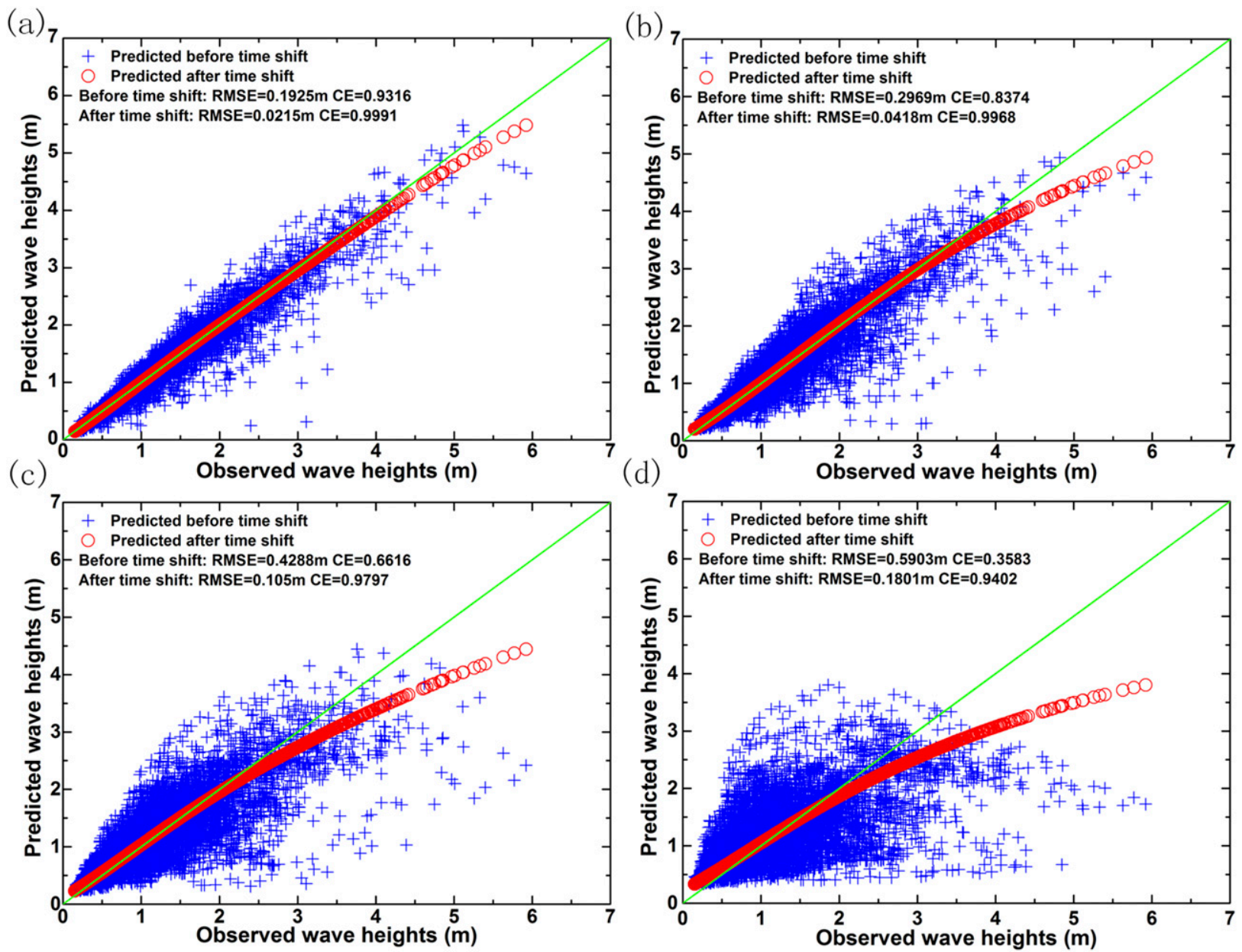

FIG. 11. Comparisons of the prediction effects between before and after the time shift for 42001: lead time (a) 3, (b) 6, (c) 12, and (d) $24 \mathrm{~h}$.

where $b \_\operatorname{star}_{i}$ and $c \_s t a r_{j}$ are the dynamic biases for the visible unit $i$ and hidden one $j ; v_{k}^{t-q}$ is the data for the visible unit $k$ at time $t-q ; \alpha_{k i}^{t-q}$ is the directed connection weight between the visible unit $k$ and $i$ while $\beta_{k j}^{t-q}$ is the one between the visible unit $k$ and the hidden one $j$; and $b_{i}$ and $c_{j}$ are the static biases of the visible unit $i$ and hidden one $j$.

\section{c. $P S O$}

Key model parameters, for example, the number of neurons in the input layer, the number of hidden layers, the number of neurons in every hidden layer and their combination, directly influence training results and predictive accuracy. In this paper, the particle swarm optimization algorithm, which has the advantages of a simple and fast search and is suitable for real-valued data processing, is adopted to determine key model parameters.

PSO, which was derived from the study of social behavior of bird flocking, was first proposed by Kennedy and Eberhart (1995). Each particle in the algorithm, which is corresponding to a fitness value determined by the fitness function, represents a potential solution for the problem. The velocity of a particle determines its moving direction and distance, adjusts dynamically and empirically with movements of it and other particles, and thus realizes the optimization in the solvable space. In this paper, key model parameters are regarded as particles. The mean square error (MSE) between the CRBM-DBN output (i.e., the predicted value) and the label (i.e., the observed value) is designated as the fitness function. The best key model parameters can be determined by minimizing the MSE. The concrete procedure of PSO is as follows:

1) Initial positions and velocities of key model parameters.

2) Compute MSEs for key model parameters.

3) Search individual and integral best MSEs for key model parameters.

4) Update velocities and positions of key model parameters according to Eqs. (16) and (17): 

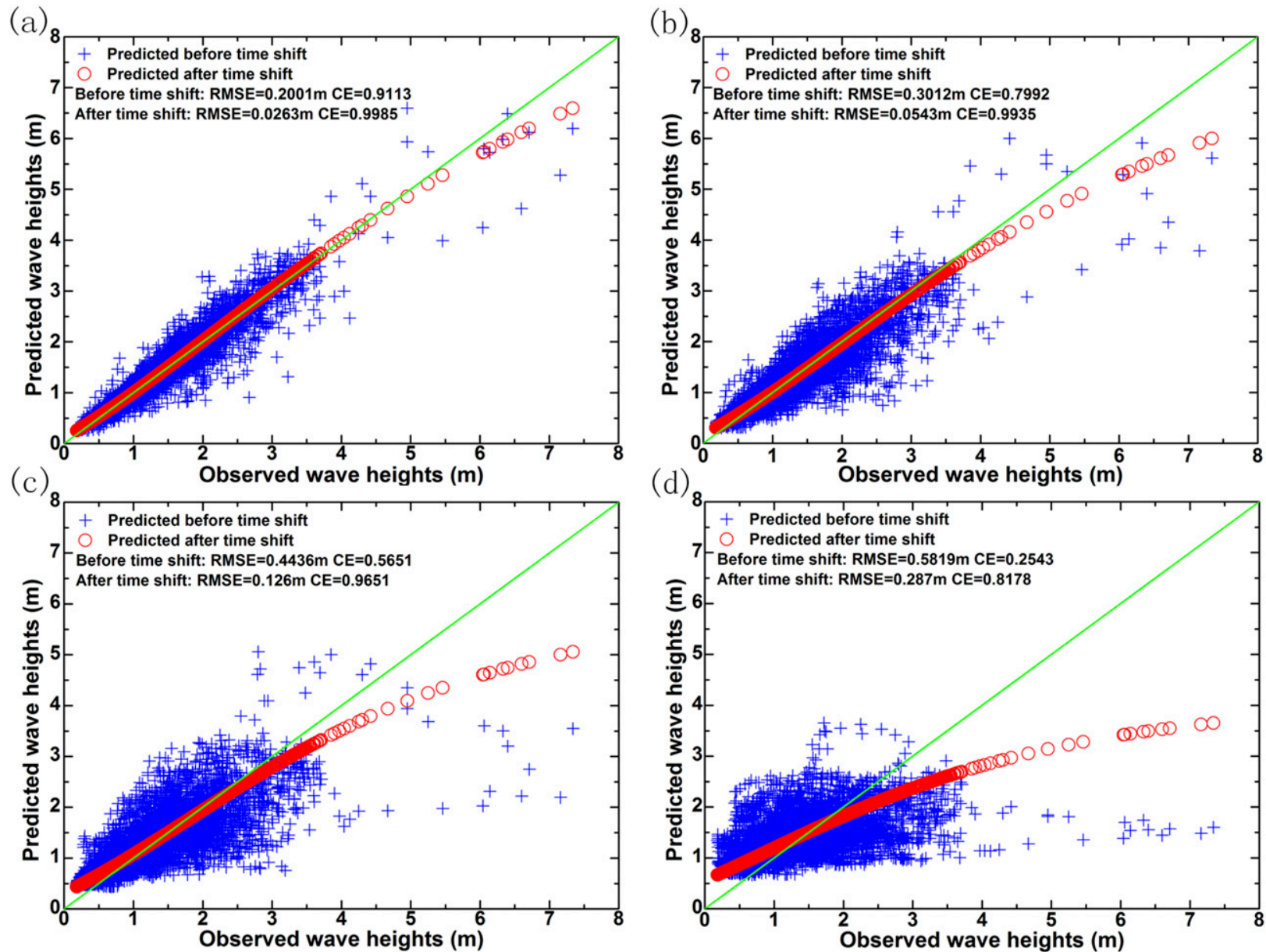

FIG. 12. Comparisons of the prediction effects between before and after the time shift for 42020: lead time (a) 3, (b) 6, (c) 12, and (d) $24 \mathrm{~h}$.

$$
V_{i}^{k+1}=\omega V_{i}^{k}+c_{1} r_{1}\left(P_{i}^{k}-X_{i}^{k}\right)+c_{2} r_{2}\left(P_{g}^{k}-X_{i}^{k}\right),
$$

and

$$
X_{i}^{k+1}=X_{i}^{k}+V_{i}^{k+1},
$$

where $\omega$ is inertia weight, $k$ is current iteration times, $V_{i}$ is the velocity for the $i$ th key model parameter, $X_{i}$ is the position for the $i$ th key model parameter, acceleration factors $c_{1}$ and $c_{2}$ are nonnegative constants, $r_{1}$ and $r_{2}$ are random numbers distributed in [0,1], and $P_{i}$ and $P_{g}$ represent individual and integral best fitness values for key model parameters, respectively. Velocities and positions of key model parameters are limited within a certain range so as to prevent their blind search.

5) If stopping criteria, for example, iteration times are unsatisfied, then return to step 2 and continue; otherwise, to the end.

\section{d. CRBM-DBN prediction model}

With Fig. 2 and Fig. 4 combined and RBM substituted to CRBM, the CRBM-DBN prediction model is illustrated as Fig. 5.

Similar to the RBM stacking, the CRBM-DBN model constructed in Fig. 5 stacks multilayer CRBMs. The training process of the CRBM-DBN is also similar to the one of DBN. Only in the pretraining are dynamic updates for biases required to be added because of the existence of AR connections between the historical time and the current one.

The CRBM-DBN training process is described as follows:

Stage 1: Unsupervised pretraining

1) Weights and biases in the CRBM-DBN model are initialized to 0 .

2) Adjacent two layers in the model are recognized as one CRBM, the model is trained with the CD algorithm layer by layer, and parameters are 
(a)

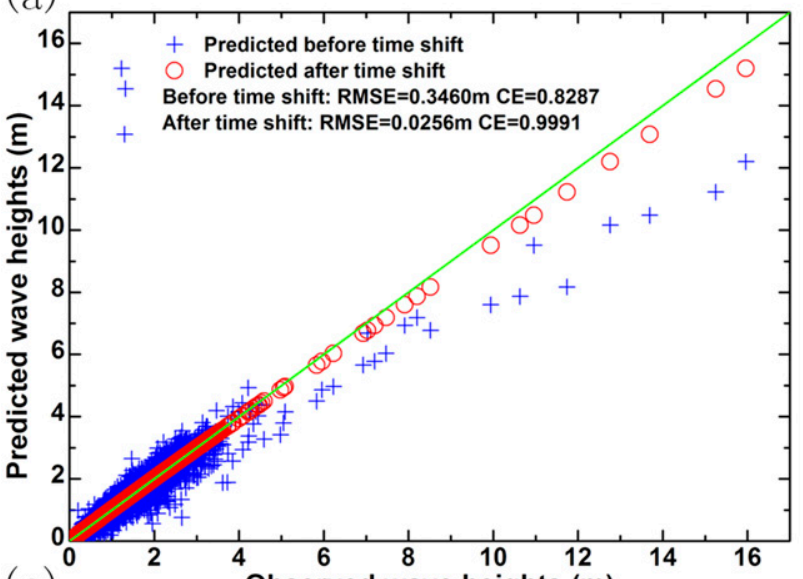

(c)

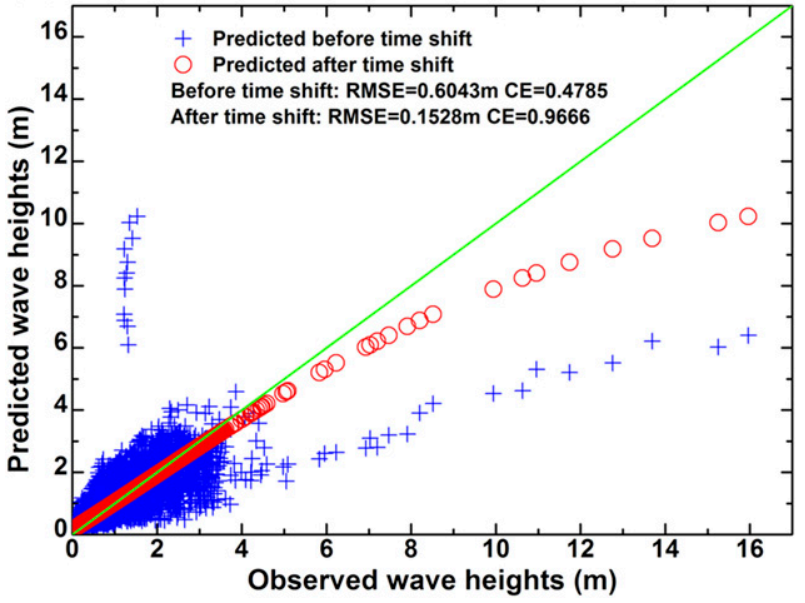

(b)

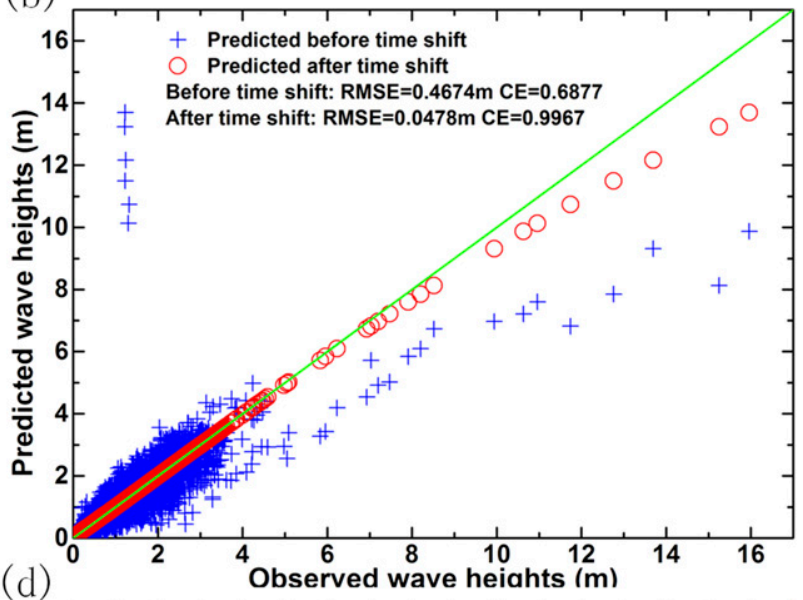

(d)

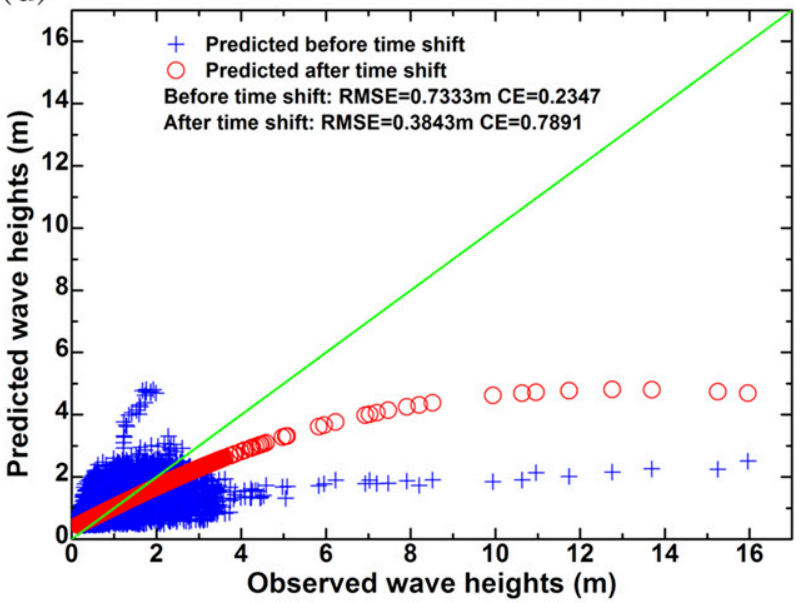

FIG. 13. Comparisons of the prediction effects between before and after the time shift for 42040: lead time (a) 3, (b) 6, (c) 12, and (d) $24 \mathrm{~h}$.

updated. Specifically, biases of units are dynamically updated.

Stage 2: Supervised fine tuning

1) Pretrained model parameters are used as initial values.

2) Parameters of the CRBM-DBN are fine-tuned by supervised algorithms, for example, the backward propagation algorithm.

Based on the CRBM-DBN model, with key model parameters determined by PSO, the schematic illustration for the wave height prediction is shown in Fig. 6.

\section{Study area and data}

\section{a. Study area}

Wave height data in the Gulf of Mexico shared by the NDBC are used as study objects in this paper. Specifically, wave height data from the nearshore buoy (ID 42020) and offshore buoy (ID 42001) in 2016 are selected as training data and used for determining model parameters, respectively. Accordingly, wave height data of the two buoys in 2017 are taken as testing data. Furthermore, for investigating the prediction effect on an extreme event, the data of the buoy with ID 42040 in 2003 are trained and the data in 2004 are tested since the huge wave heights caused by Hurricane Ivan in 2004 were observed. Predicted and observed values are compared, and the prediction accuracies for the three buoys are evaluated. Figure 7 shows the study area. Exact locations, water depths, and data characteristics for 42001, 42020, and 42040 are displayed in Table 1 .

\section{b. Data preprocessing}

Before the unsupervised layer-wise pretraining, wave height data for training are required to deal with Gaussian normalization due to the CRBM existence. Namely, training data are normalized to have zero mean and unit variance according to Eq. (18): 


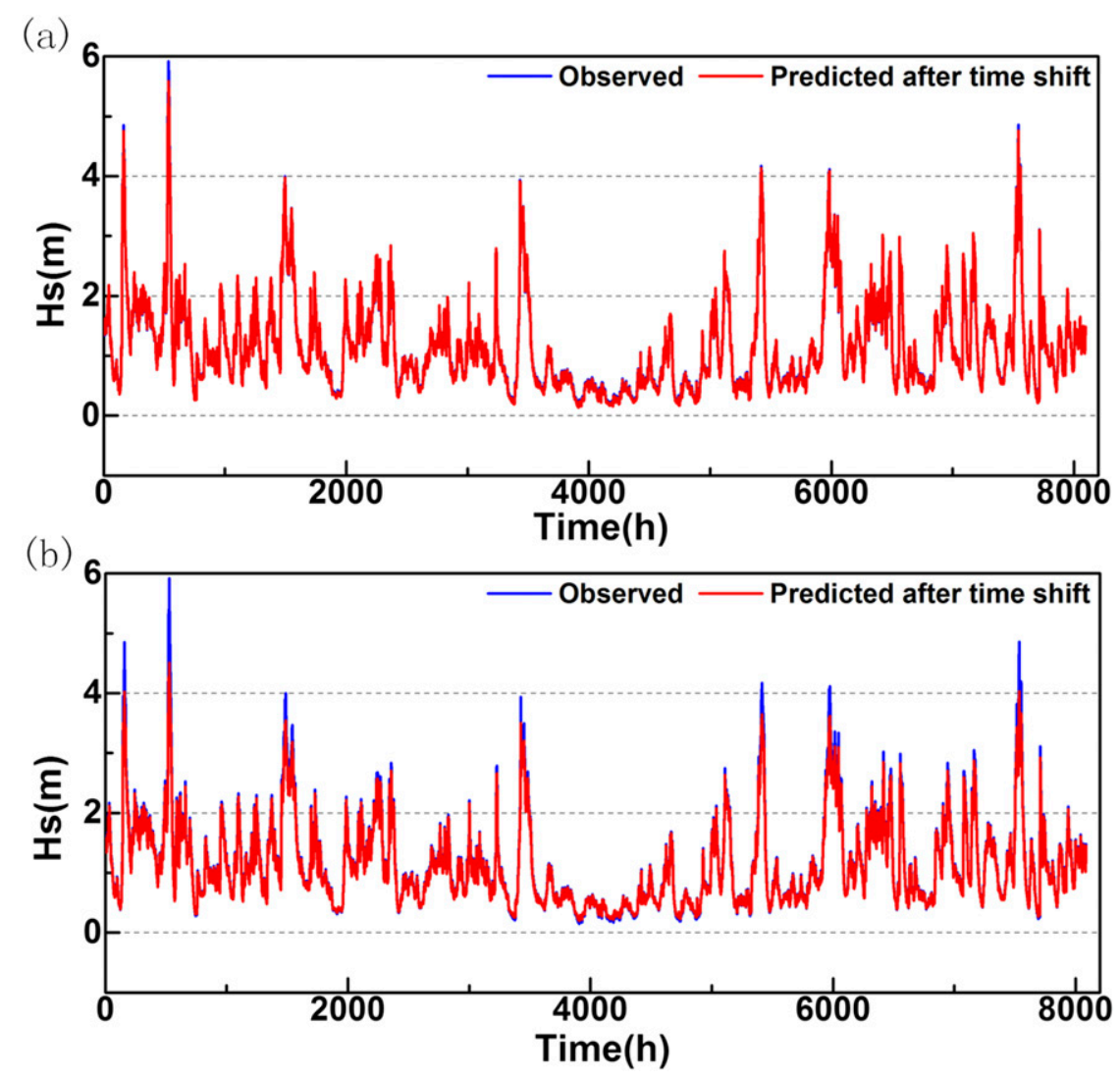

FIG. 14. Observed and predicted time series of 42001: lead time (a) 1 and (b) $9 \mathrm{~h}$.

$$
y^{*}=\frac{y-y_{\text {mean }}}{y_{\text {std }}}
$$

where $y$ and $y^{*}$ are before and after normalized training data and $y_{\text {mean }}$ and $y_{\text {std }}$ represent the mean and standard error of training data, respectively.

Before the supervised backward fine tuning, training data are needed to normalize to $[0,1]$ on the basis of Eq. (19):

$$
y^{*}=\frac{y-y_{\min }}{y_{\max }-y_{\min }},
$$

where $y_{\max }$ and $y_{\min }$ represent the maximum and minimum of training data, respectively. Testing data are required to preprocess in the same way before the prediction.

\section{c. Evaluation criterion}

The model prediction accuracy depends to some extent on the selection of the evaluation criterion. Kim and Park (2005) pointed out that although the correlation coefficient is widely used in various analyses of engineering data, it is more suitable for assessing the linearity between two datasets and not for the agreement between predicted and observed values. We also find from wave height data analysis that larger differences may exist when higher correlation coefficients appear. Nash-Sutcliffe coefficient of efficiency (CE) and rootmean-square error (RMSE) therefore are used to evaluate the prediction ability of the model in the paper. CE and RMSE can refer to Eqs. (20) and (21):

$$
\mathrm{CE}=1-\frac{\sum_{i=1}^{N}(X-Y)^{2}}{\sum_{i=1}^{N}(X-\bar{X})^{2}},
$$

and

$$
\mathrm{RMSE}=\sqrt{\frac{\sum_{i=1}^{N}(X-Y)^{2}}{N},}
$$

where $X$ and $Y$ are the actual observed and predicted values for wave height and $\bar{X}$ is the mean value of $X$. Term $N$ is the number of wave height data.

Moreover, we employ the relative error to evaluate the prediction effect for the maximum wave 

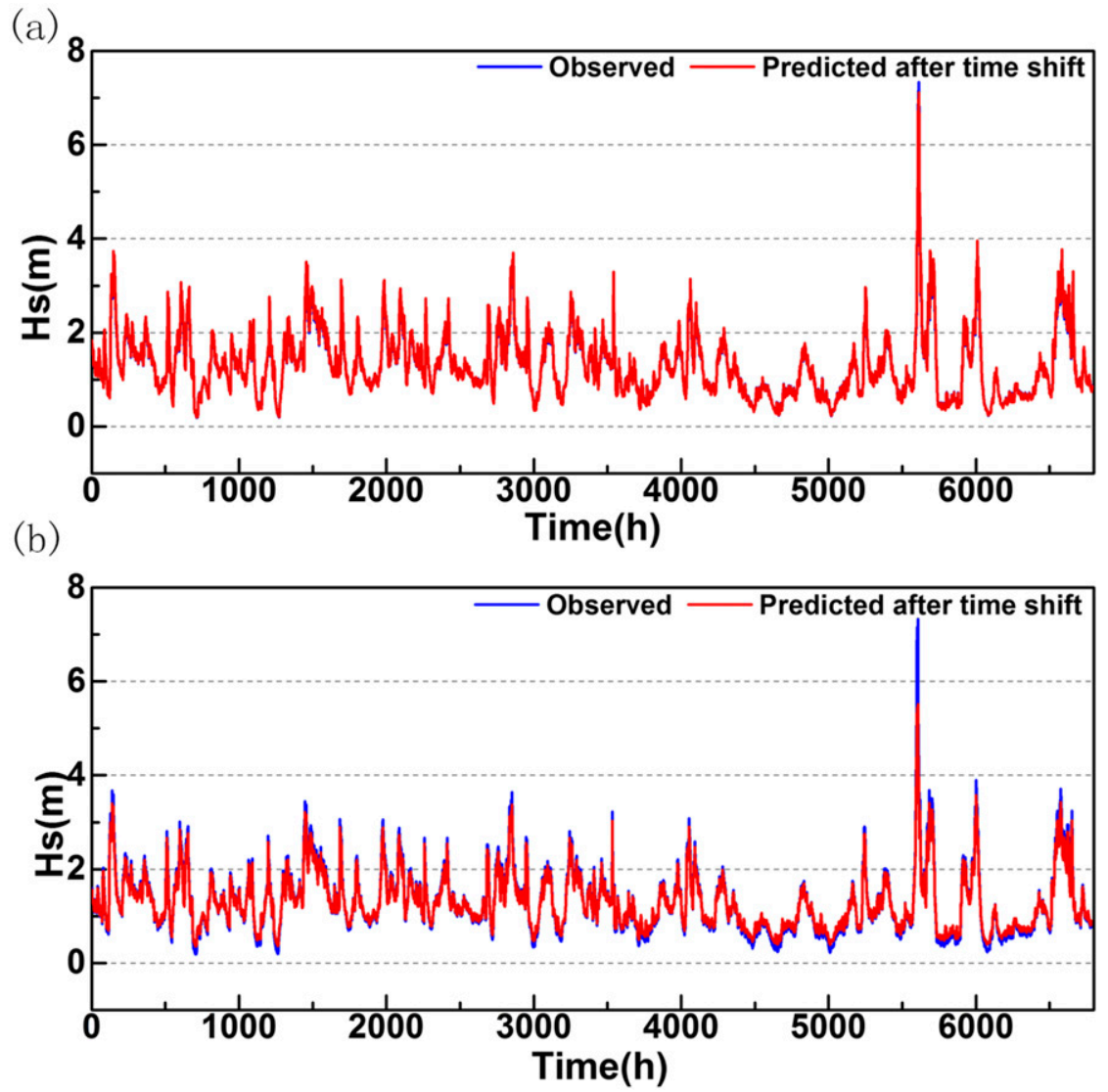

FIG. 15. Observed and predicted time series of 42020: lead time (a) 1 and (b) $9 \mathrm{~h}$.

height. Equation (22) for the relative prediction error $(\mathrm{RPE})$ is

$$
\mathrm{RPE}=\frac{(Y-X)}{X} \times 100 \% .
$$

\section{Experiments}

\section{a. Model parameters}

From the prediction schematic illustration in Fig. 6, PSO is used to determine CRBM-DBN model parameters for wave height training data of 42001 and 42020 in 2016 and 42040 in 2003. Experiments indicate that excessive model parameters may lead to a very long search process. From the influence of parameters to prediction results point of view, key model parameters, which are the number of neurons in the input layer, the number of hidden layers, and the number of neurons in each hidden layer, are determined by PSO. To improve the efficiency the search scope for the number of neurons in the input layer is limited to $[1,6]$ based on experiences. Similarly, the number of hidden layers and the number of neurons in each hidden layer are bounded to $[1,5]$ and $[3,10]$, respectively.

Table 2 shows the best structure parameters by PSO searching, which are marked with bold font, and other ones used in the model for 42001, 42020, and 42040.

\section{b. Predicted results}

According to the CRBM-DBN training procedure, model parameters in Table 2 are employed, and the wave height testing data of 42001 and 42020 in 2017 and 42040 in 2004 are used to predict. The lead times for forecasting are $1,3,6,9,12$, and $24 \mathrm{~h}$. In other words, supposing $t$ is the current moment, the observed wave height at times $t-3, t-2, t-1$, and $t$ are taken as the input for 42001 and 42040 and at times $t-2, t-1$, and $t$ for 42020. The wave height output at times $t+1$, $t+3, t+6, t+9, t+12$, and $t+24$ for the three buoys are predicted. It should be pointed out that the prediction curves lag the lead time behind the observed ones fully and regularly from all of the forecasting results. Partial predictive results of 42040 with lead times of 1 and $3 \mathrm{~h}, 42020$ with lead times of 6 and $9 \mathrm{~h}$, and 42001 with lead times of 12 and $24 \mathrm{~h}$ are shown in 

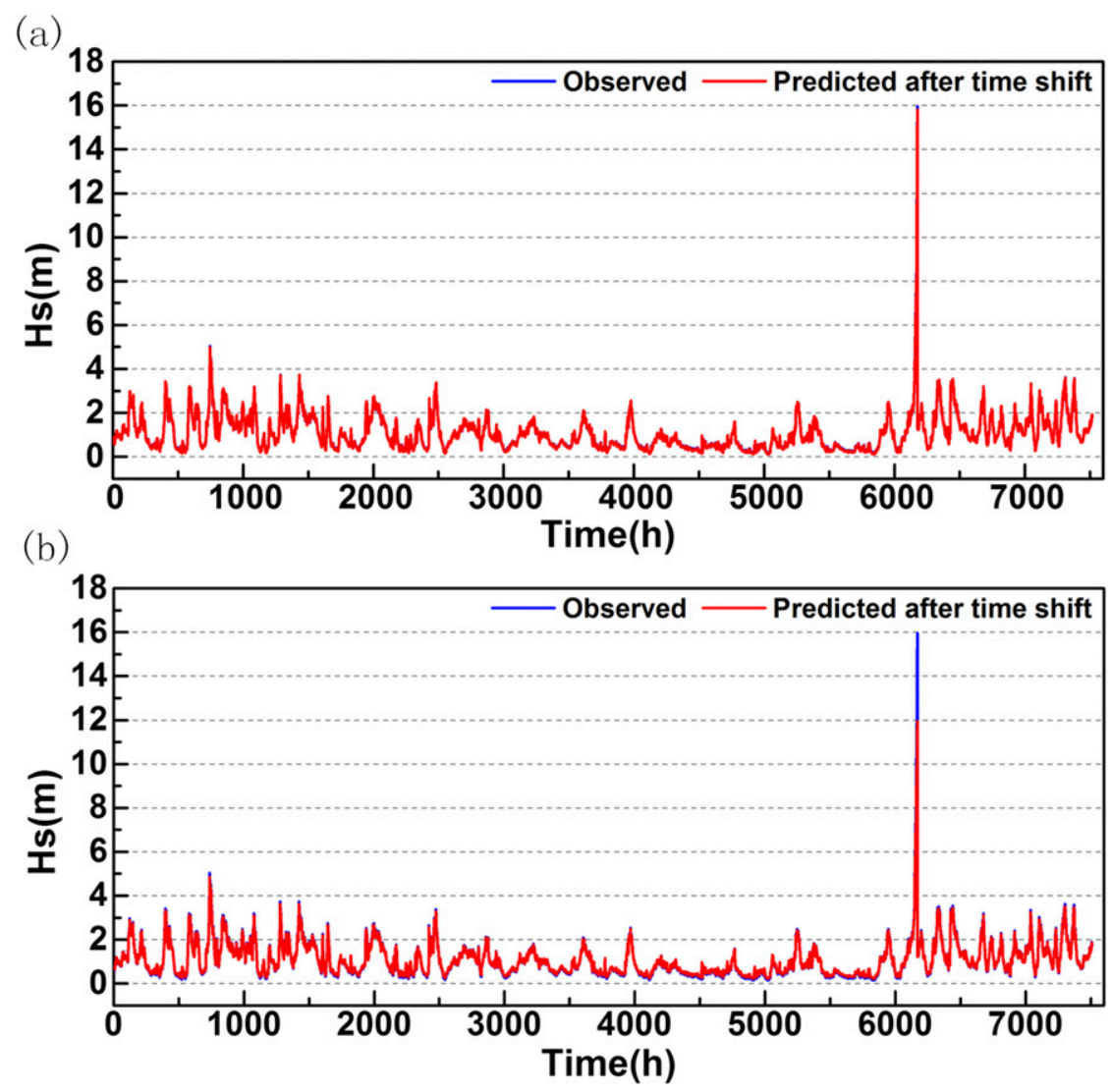

FIG. 16. Observed and predicted time series of 42040: lead time (a) 1 and (b) $9 \mathrm{~h}$.

Figs. 8-10, where the black dot-dashed line represents the observed time series while the blue line stands for the prediction.

Comparing the black dot-dashed line with the blue line in Figs. 8-10, the regular prediction lags exist in the prediction curves with different lead times. Similar to the lag phenomenon of the artificial neural network prediction, this may be because the CRBM-DBN model also gives the most weight to the latest input at $t$ for calculating the forecast output at $t+$ lead time (de Vos and Rientjes 2005). Consequently, referring to the time shift operation in Abrahart et al. (2007), we also employ the timing correction factor (i.e., lead time) to shift the original prediction results, and the predictive curves after the time shift are illustrated as the red lines in Figs. 8-10. The lags are removed completely after the prediction curves shifting by lead time. Compared to the prediction curves before the time shift, the prediction curves after the time shift coincide with the observed curves obviously.

With the predictions of lead times of $3,6,12$, and $24 \mathrm{~h}$ for 42001, 42020, and 42040 as examples, Figs. 11-13 compare the prediction effects before and after the time shifting by scatter diagrams.
Figures 11-13 compare separately the prediction scatter diagram after the time shifting converges to the perfect model line ( $45^{\circ}$ line) more obviously relative to the one before the time shifting. Naturally, the

TABLE 3. Prediction accuracy specifications of wave height for 42001, 42020, and 42040 with different lead times.

\begin{tabular}{lccc}
\hline \hline ID & Lead time (h) & CE & RMSE (m) \\
\hline 42001 & 1 & 0.9995 & 0.0172 \\
& 3 & 0.9991 & 0.0215 \\
6 & 0.9968 & 0.0418 \\
& 9 & 0.9892 & 0.0764 \\
42020 & 12 & 0.9797 & 0.105 \\
& 24 & 0.9402 & 0.180 \\
& 1 & 0.9988 & 0.0237 \\
& 3 & 0.9985 & 0.0263 \\
42040 & 6 & 0.9935 & 0.0543 \\
& 9 & 0.9830 & 0.0877 \\
& 12 & 0.9651 & 0.126 \\
& 24 & 0.8178 & 0.287 \\
& 1 & 0.9995 & 0.0195 \\
& 6 & 0.9991 & 0.0256 \\
& 9 & 0.9967 & 0.0478 \\
& 12 & 0.9875 & 0.0934 \\
& 24 & 0.9666 & 0.1528 \\
& & 0.7891 & 0.3843 \\
\hline
\end{tabular}


TABLE 4. Maximum wave height prediction results for 42001, 42020, and 42040 with lead times of 1,3 , and $6 \mathrm{~h}$. "Obs" is observed values and "Pre" is predicted values.

\begin{tabular}{|c|c|c|c|c|c|c|c|}
\hline \multirow[b]{2}{*}{ ID } & \multirow[b]{2}{*}{ Obs (m) } & \multicolumn{2}{|c|}{ Lead time $1 \mathrm{~h}$} & \multicolumn{2}{|c|}{ Lead time $3 \mathrm{~h}$} & \multicolumn{2}{|c|}{ Lead time $6 \mathrm{~h}$} \\
\hline & & Pre (m) & RPE & Pre $(m)$ & $\mathrm{RPE}$ & Pre $(m)$ & RPE \\
\hline \multirow[t]{3}{*}{42001} & 5.77 & 5.49 & $-4.9 \%$ & 5.38 & $-6.8 \%$ & 4.86 & $-15.8 \%$ \\
\hline & 5.92 & 5.59 & $-5.6 \%$ & 5.48 & $-7.4 \%$ & 4.93 & $-16.7 \%$ \\
\hline & 5.63 & 5.38 & $-4.3 \%$ & 5.27 & $-6.3 \%$ & 4.79 & $-15.0 \%$ \\
\hline \multirow[t]{3}{*}{42020} & 7.16 & 6.97 & $-2.6 \%$ & 6.48 & $-9.4 \%$ & 5.91 & $-17.5 \%$ \\
\hline & 6.71 & 6.60 & $-1.7 \%$ & 6.19 & $-7.7 \%$ & 5.67 & $-15.5 \%$ \\
\hline & 7.34 & 7.12 & $-3.0 \%$ & 6.60 & $-10.1 \%$ & 6.00 & $-18.3 \%$ \\
\hline \multirow[t]{3}{*}{42040} & 15.25 & 15.12 & $-0.86 \%$ & 14.54 & $-4.64 \%$ & 13.23 & $-13.23 \%$ \\
\hline & 13.69 & 13.53 & $-1.12 \%$ & 13.08 & $-4.46 \%$ & 12.17 & $-11.14 \%$ \\
\hline & 15.96 & 15.83 & $-0.83 \%$ & 15.20 & $-4.79 \%$ & 13.70 & $-14.19 \%$ \\
\hline
\end{tabular}

prediction accuracy has been greatly improved according to the variations of CE and RMSE.

As examples, Figs. 14-16 exhibit the observed and predicted time series of 42001, 42020, and 42040 for 1 and 9 -h predictions.

It can be found in Figs. 14a, 15a, and 16a that for the nearshore buoys 42020 and 42040 and the offshore buoy 42001 , when the lead time is $1 \mathrm{~h}$, the predicted values conform correctly to the observed ones pertaining to the wave height inherent trend. Besides, the CRBM-DBN model performs extremely well for the predictions of the maximum wave heights, especially for the extreme event observed by 42040 . When the lead time increases to $9 \mathrm{~h}$ (Figs. 14b, 15b, 16b), deviations between predicted and observed values are more obvious, especially for the waves with more than 3-m heights. In fact, the red curves in Figs. 11-13 deviate gradually from the green perfect $45^{\circ}$ line, and the whole prediction effects for the three buoys are all become worse with the lead time increasing.

The wave height forecasting accuracies of the three buoys for lead times $1-24 \mathrm{~h}$ are illustrated in Table 3.

Table 3 shows that for the offshore buoy 42001, RMSE increases from $\sim 2$ to $\sim 20 \mathrm{~cm}$ while CE decreases from 0.9995 to 0.9402 with the lead time increasing. The prediction effects for the coastal buoys 42020 and 42040 are a little bit worse than 42001 . When the lead time is less than or equal to $12 \mathrm{~h}, \mathrm{RMSE}$ is less than $\sim 0.16 \mathrm{~m}$ and CE is more than $\sim 0.96$. When the lead time is equal to $24 \mathrm{~h}$, RMSE is $0.287 \mathrm{~m}$ and CE is 0.8178 for 42020 while the forecast error of 42040 , that is, RMSE is $0.3843 \mathrm{~m}$ and CE is 0.7891 , is slightly larger due to the existence of the extraordinary extreme event.

Tables 4 and 5 show the prediction results of maximum wave height for 42001, 42020, and 42040 with different lead times.

According to Tables 4 and 5, similar to the whole prediction error, the prediction error of the maximum wave height is also increasing as the lead time increases. With regard to maximum wave heights of the three buoys, the predicted wave heights are all lower than the observed ones. The greater the measured wave height, the larger the prediction error for each buoy. For 42001 and 42020 , the ranges of RPE are [4.3\%, 35.7\%] and $[1.7 \%, 50.2 \%]$, respectively, while the RPE of the huge wave heights resulting from Hurricane Ivan increase from $0.83 \%$ to $70.58 \%$.

TABLE 5. Maximum wave height prediction results for 42001, 42020, and 42040 with lead times of 9, 12 , and $24 \mathrm{~h}$. "Obs" is observed values and "Pre" is predicted values.

\begin{tabular}{|c|c|c|c|c|c|c|c|}
\hline \multirow[b]{2}{*}{ ID } & \multirow[b]{2}{*}{ Obs (m) } & \multicolumn{2}{|c|}{ Lead time $9 \mathrm{~h}$} & \multicolumn{2}{|c|}{ Lead time $12 \mathrm{~h}$} & \multicolumn{2}{|c|}{ Lead time $24 \mathrm{~h}$} \\
\hline & & Pre $(\mathrm{m})$ & RPE & Pre $(m)$ & RPE & Pre $(\mathrm{m})$ & RPE \\
\hline \multirow[t]{3}{*}{42001} & 5.77 & 4.45 & $-22.9 \%$ & 4.37 & $-24.2 \%$ & 3.76 & $-34.9 \%$ \\
\hline & 5.92 & 4.51 & $-23.8 \%$ & 4.44 & $-24.9 \%$ & 3.80 & $-35.7 \%$ \\
\hline & 5.63 & 4.39 & $-22.0 \%$ & 4.30 & $-23.6 \%$ & 3.71 & $-34.1 \%$ \\
\hline \multirow[t]{3}{*}{42020} & 7.16 & 5.45 & $-23.9 \%$ & 5.00 & $-30.1 \%$ & 3.63 & $-49.4 \%$ \\
\hline & 6.71 & 5.26 & $-21.6 \%$ & 4.86 & $-27.6 \%$ & 3.55 & $-47.1 \%$ \\
\hline & 7.34 & 5.52 & $-24.8 \%$ & 5.06 & $-31.1 \%$ & 3.65 & $-50.2 \%$ \\
\hline \multirow[t]{3}{*}{42040} & 15.25 & 11.66 & $-23.55 \%$ & 10.03 & $-34.24 \%$ & 4.74 & $-68.90 \%$ \\
\hline & 13.69 & 10.94 & $-20.09 \%$ & 9.53 & $-30.41 \%$ & 4.80 & $-64.92 \%$ \\
\hline & 15.96 & 11.95 & $-25.10 \%$ & 10.23 & $-35.90 \%$ & 4.70 & $-70.58 \%$ \\
\hline
\end{tabular}


TABLE 6. Prediction error statistics for 42001, 42020, and 42040 with different training durations.

\begin{tabular}{|c|c|c|c|c|c|c|c|c|}
\hline \multirow[b]{2}{*}{ ID } & \multirow[b]{2}{*}{ Training duration } & \multirow[b]{2}{*}{ Prediction error } & \multicolumn{6}{|c|}{ Lead time } \\
\hline & & & $1 \mathrm{~h}$ & $3 \mathrm{~h}$ & $6 \mathrm{~h}$ & $9 \mathrm{~h}$ & $12 \mathrm{~h}$ & $24 \mathrm{~h}$ \\
\hline \multirow[t]{4}{*}{42001} & \multirow[t]{2}{*}{ 2015-16 } & RMSE (m) & 0.0157 & 0.0297 & 0.0715 & 0.1261 & 0.1839 & 0.3204 \\
\hline & & $\mathrm{CE}$ & 0.9995 & 0.9984 & 0.9906 & 0.9706 & 0.9376 & 0.8108 \\
\hline & \multirow[t]{2}{*}{$2012-16$} & RMSE (m) & 0.0179 & 0.0384 & 0.0868 & 0.1405 & 0.2015 & 0.3875 \\
\hline & & $\mathrm{CE}$ & 0.9994 & 0.9973 & 0.9861 & 0.9636 & 0.9251 & 0.7234 \\
\hline \multirow[t]{4}{*}{42020} & \multirow[t]{2}{*}{ 2015-16 } & RMSE (m) & 0.013 & 0.0167 & 0.0374 & 0.09 & 0.121 & 0.2559 \\
\hline & & $\mathrm{CE}$ & 0.9996 & 0.9994 & 0.9969 & 0.9821 & 0.9676 & 0.8553 \\
\hline & \multirow[t]{2}{*}{$2012-16$} & RMSE (m) & 0.0128 & 0.02 & 0.0668 & 0.1088 & 0.1521 & 0.3568 \\
\hline & & $\mathrm{CE}$ & 0.9996 & 0.9991 & 0.9901 & 0.9738 & 0.9488 & 0.7186 \\
\hline \multirow[t]{4}{*}{42040} & \multirow[t]{2}{*}{$2002-03$} & RMSE (m) & 0.0342 & 0.0456 & 0.113 & 0.1511 & 0.185 & 0.6717 \\
\hline & & $\mathrm{CE}$ & 0.9983 & 0.997 & 0.9817 & 0.9673 & 0.951 & 0.3558 \\
\hline & \multirow[t]{2}{*}{ 1999-2003 } & RMSE (m) & 0.0366 & 0.0488 & 0.1018 & 0.2025 & 0.3202 & 0.9905 \\
\hline & & $\mathrm{CE}$ & 0.9981 & 0.9966 & 0.9852 & 0.9413 & 0.8534 & -0.401 \\
\hline
\end{tabular}

It is known from the prediction results of the three buoys with different lead times that the overall effect of the CRBM-DBN model for the short-term ( $\leq 9 \mathrm{~h})$ wave height forecasting is excellent. Even for the extreme event observed by 42040 , the short-term prediction effect of the model is also satisfactory. It may be attributed to the superior ability of the CRBM-DBN model constructing the relationships among the shortterm wave heights and the feature extraction. With the lead time increasing, the correlation of the wave heights with the previous wave characteristics becomes lower in larger lead times. Therefore, the long-term $(>9 \mathrm{~h})$ prediction accuracy needs to improve, especially for the nearshore buoys. In addition, compared with 42001, the prediction effects of CRBM-DBN for 42020 and 42040 are relatively poorer in the same conditions. It may be because the topographic conditions and wave generation mechanism for coastal areas are more complicated than open seas.

To reveal the relationship between the prediction error and the training duration, we have extended the training time and investigated the forecasting performance of the model. For example, for 42001, the training duration increases to 2 years, that is, the wave height data in 2015 and 2016 are trained and the data in
2017 are tested. It can be seen from Table 6 that as a whole, the prediction effects of 42001 and 42040 descend with the training time getting longer. For 42020 , the whole prediction effect with the training duration of 2 years is superior to the one trained with 1 year of historical data. However, the prediction error with a lead time of more than $6 \mathrm{~h}$ has become larger when the training duration extends from 2 to 5 years. Consequently, it seems that the relationship between the prediction error and the training duration is not clear for the buoys in the paper.

\section{c. Algorithms comparison}

To compare the short-term prediction effect between CRBM-DBN and other algorithms, we select the classic autoregressive-moving-average (ARMA) model and the BP neural network, which is used widely in shallow machine learning and the traditional DBN, that is, RBM-DBN in deep machine learning. With different lead times, Tables 7-9 list prediction results of four models for the three buoys. Based on the method in the literature (Agrawal and Deo 2002), $\operatorname{ARMA}(1,1)$ is used to predict the wave height in the paper. From the forecasting results of ARMA and BP in Tables 7-9, the BP neural network has distinct

TABLE 7. Short-term prediction accuracy comparisons of four models for 42001.

\begin{tabular}{|c|c|c|c|c|c|}
\hline Lead time & Evaluation criteria & ARMA & $\mathrm{BP}$ & RBM-DBN & CRBM-DBN \\
\hline \multirow[t]{2}{*}{$1 \mathrm{~h}$} & $\mathrm{CE}$ & 0.9782 & 0.986 & 0.9988 & 0.9995 \\
\hline & RMSE (m) & 0.1086 & 0.0946 & 0.0253 & 0.0172 \\
\hline \multirow[t]{2}{*}{$3 \mathrm{~h}$} & $\mathrm{CE}$ & 0.9317 & 0.979 & 0.9975 & 0.9991 \\
\hline & RMSE (m) & 0.1923 & 0.0987 & 0.037 & 0.0215 \\
\hline \multirow[t]{2}{*}{$6 \mathrm{~h}$} & $\mathrm{CE}$ & 0.8407 & 0.9612 & 0.9903 & 0.9968 \\
\hline & RMSE (m) & 0.2937 & 0.121 & 0.0726 & 0.0418 \\
\hline \multirow[t]{2}{*}{$9 \mathrm{~h}$} & $\mathrm{CE}$ & 0.747 & 0.9598 & 0.9833 & 0.9892 \\
\hline & RMSE (m) & 0.3701 & 0.167 & 0.0952 & 0.0764 \\
\hline
\end{tabular}


TABLE 8. Short-term prediction accuracy comparisons of four models for 42020.

\begin{tabular}{cccccc}
\hline \hline Lead time & Evaluation criteria & ARMA & BP & RBM-DBN & CRBM-DBN \\
\hline \multirow{2}{*}{$1 \mathrm{~h}$} & CE & 0.9699 & 0.981 & 0.9978 & 0.9988 \\
& RMSE (m) & 0.1166 & 0.101 & 0.0317 & 0.0237 \\
$\mathrm{~h}$ & CE & 0.9134 & 0.9745 & 0.9959 & 0.9985 \\
& RMSE (m) & 0.1976 & 0.118 & 0.0429 & 0.0263 \\
$\mathrm{~h}$ & CE & 0.7993 & 0.9673 & 0.9866 & 0.9935 \\
& RMSE (m) & 0.3009 & 0.156 & 0.0843 & 0.0543 \\
$\mathrm{~h}$ & CE & 0.6737 & 0.9401 & 0.9692 & 0.983 \\
& RMSE (m) & 0.3837 & 0.187 & 0.118 & 0.0877 \\
\hline
\end{tabular}

superiority over the $\operatorname{ARMA}(1,1)$ method in the short-term prediction as with the conclusion of Agrawal and Deo (2002). Comparing the RMSE and CE of BP, RBM-DBN, and CRBM-DBN in Tables 7-9, we find that for the same lead time, the prediction accuracies of RBM-DBN and CRBM-DBN are apparently higher than the BP model since some deficiencies of shallow learning are made up by deep learning. The prediction accuracy of the CRBMDBN model, which considers the continuity of time series data and constructs AR connections between historical and current data, is superior to RBMDBN.

\section{Conclusions}

The CRBM-DBN model is used to predict the wave height in the paper. The wave heights observed by 42001 and 42020 in 2016 and 42040 in 2003 are taken as training data. PSO is employed to determine key model parameters. The wave data in 2017 for 42001 and 42020 and the data in 2004 for 42040 are tested with the wave-wave pattern. The lead time is selected as $1,3,6,9,12$, or $24 \mathrm{~h}$. CE and RMSE are used as the evaluation criteria for the whole prediction error. With the lead time increasing, as a whole, the prediction error of CRBM-DBN for the wave height enlarges from 1-2 to $\sim 40 \mathrm{~cm}$ for RMSE while it decreases from 0.9995 to 0.7891 for CE. The overall error is less than $10 \mathrm{~cm}$ (RMSE) and more than 0.98 (CE) for the short-term $(\leq 9 \mathrm{~h})$ prediction. Predicted values are all underobserved for maximum wave heights, and the relative prediction error increases from $0.83 \%$ to $25.10 \%$ when the lead time increases to $9 \mathrm{~h}$. It is demonstrated by comparisons with prediction results of ARMA, BP, and RBM-DBN models that the CRBMDBN model in the paper has better short-term forecasting ability. What needs to be pointed out is that the results in the paper are based on the observed data of 42040, 42001, and 42020 in the Gulf of Mexico. In view of regional differences among oceans, if buoys in other regions are regarded as objects, it may achieve the ideal prediction accuracy by following the method in the paper and adjusting the parameters of the CRBM-DBN model.

Acknowledgments. The authors thank the support from the National Nature Science Foundation for Young Scientists of China (Grant No. 61505056). All significant wave height data used in this study were obtained from https://www.ndbc.noaa.gov/station history.php?station $=42040$, https://www.ndbc.noaa. gov/station_history.php?station $=42001$, and https:// www.ndbc.noaa.gov/station_history.php?station $=42020$. Special thanks for the data support from U.S. National Data Buoy Center and the positive suggestions from Engineer Famei Lei of Xiamen University. We are indebted to two anonymous reviewers who provided extensive comments and suggestions to help improve this manuscript.

TABLE 9. Short-term prediction accuracy comparisons of four models for 42040.

\begin{tabular}{cccccc}
\hline \hline Lead time & Evaluation criteria & ARMA & BP & RBM-DBN & CRBM-DBN \\
\hline \multirow{2}{*}{$1 \mathrm{~h}$} & CE & 0.939 & 0.974 & 0.998 & 0.9995 \\
& RMSE (m) & 0.2065 & 0.116 & 0.0289 & 0.0195 \\
$3 \mathrm{~h}$ & CE & 0.8296 & 0.971 & 0.9949 & 0.9991 \\
& RMSE (m) & 0.345 & 0.131 & 0.0465 & 0.0256 \\
$6 \mathrm{~h}$ & CE & 0.6801 & 0.967 & 0.9882 & 0.9967 \\
& RMSE (m) & 0.4727 & 0.154 & 0.0816 & 0.0478 \\
$\mathrm{~h}$ & CE & 0.5523 & 0.9349 & 0.9607 & 0.9875 \\
& RMSE (m) & 0.5592 & 0.193 & 0.1215 & 0.0934 \\
\hline
\end{tabular}




\section{REFERENCES}

Abrahart, R. J., A. J. Heppenstall, and L. M. See, 2007: Timing error correction procedure applied to neural network rainfallrunoff modelling. Hydrol. Sci. J., 52, 414-431, https://doi.org/ 10.1623/hysj.52.3.414

Agrawal, J. D., and M. C. Deo, 2002: On-line wave prediction. Mar. Struct., 15, 57-74, https://doi.org/10.1016/S0951-8339(01) 00014-4.

Altunkaynak, A., and M. Özger, 2004: Temporal significant wave height estimation from wind speed by perceptron Kalman filtering. Ocean Eng., 31, 1245-1255, https://doi.org/10.1016/ j.oceaneng.2003.12.008.

Bai, Y., Z. Q. Chen, J. J. Xie, and C. Li, 2016: Daily reservoir inflow forecasting using multi-scale deep feature learning with hybrid models. J. Hydrol., 532, 193-206, https://doi.org/10.1016/ j.jhydrol.2015.11.011.

Bengio, Y., 2009: Learning deep architectures for AI. Foundations and Trends in Machine Learning, Now Publishers, 127 pp.

Booij, N., R. C. Ris, and L. H. Holthuijsen, 1999: A third-generation wave model for coastal regions: 1 . Model description and validation. J. Geophys. Res., 104, 7649-7666, https://doi.org/10.1029/ 98JC02622.

Cañellas, B., S. Balle, J. Tintoré, and A. Orfila, 2010: Wave height prediction in the Western Mediterranean using genetic algorithms. Ocean Eng., 37, 742-748, https://doi.org/10.1016/ j.oceaneng.2010.02.006.

Chen, J. F., Q. J. Jin, and J. Chao, 2012: Design of deep belief networks for short-term prediction of drought index using data in the Huaihe River basin. Math. Probl. Eng., 2012, 235929, https://doi.org/10.1155/2012/235929.

Deka, P. C., and R. Prahlada, 2012: Discrete wavelet neural network approach in significant wave height forecasting for multistep lead time. Ocean Eng., 43, 32-42, https://doi.org/ 10.1016/j.oceaneng.2012.01.017.

Deo, M. C., A. Jha, A. S. Chaphekar, and K. Ravikant, 2001: Neural networks for wave forecasting. Ocean Eng., 28, 889898, https://doi.org/10.1016/S0029-8018(00)00027-5.

Deshmukh, A. N., M. C. Deo, P. K. Bhaskaran, T. M. Balakrishnan Nair, and K. G. Sandhya, 2016: Neural-network-based data assimilation to improve numerical ocean wave forecast. IEEE J. Oceanic Eng., 41, 944-953, https://doi.org/10.1109/ JOE.2016.2521222.

de Vos, N. J., and T. H. M. Rientjes, 2005: Constraints of artificial neural networks for rainfall-runoff modelling: Trade-offs in hydrological state representation and model evaluation. Hydrol. Earth Syst. Sci., 9, 111-126, https://doi.org/10.5194/ hess-9-111-2005.

Dixit, P., and S. N. Londhe, 2016: Prediction of extreme wave heights using neuro wavelet technique. Appl. Ocean Res., 58 , 241-252, https://doi.org/10.1016/j.apor.2016.04.011.

-,- , and Y. Dandawate, 2015: Removing prediction lag in wave height forecasting using Neuro-Wavelet modeling technique. Ocean Eng., 93, 74-83, https://doi.org/10.1016/ j.oceaneng.2014.10.009.

Fischer, A., and C. Igel, 2012: An introduction to restricted Boltzmann machines. CIARP 2012: Progress in Pattern Recognition, Image Analysis, Computer Vision, and Applications, L. Alvarez et al., Eds., Lecture Notes in Computer Science, Vol. 7441, Springer, 14-36, https://doi.org/10.1007/978-3-642-33275-3_2.

Gaur, S., and M. C. Deo, 2008: Real-time wave forecasting using genetic programming. Ocean Eng., 35, 1166-1172, https://doi.org/ 10.1016/j.oceaneng.2008.04.007
Günaydın, K., 2008: The estimation of monthly mean significant wave heights by using artificial neural network and regression methods. Ocean Eng., 35, 1406-1415, https://doi.org/10.1016/ j.oceaneng.2008.07.008.

Hinton, G. E., 2002: Training products of experts by minimizing contrastive divergence. Neural Comput., 14, 1771-1800, https://doi.org/10.1162/089976602760128018.

, 2010: A practical guide to training restricted Boltzmann machines. Tech. Rep. UTML TR 2010-003, Dept. of Computer Science, University of Toronto, $20 \mathrm{pp}$.

_ , and R. R. Salakhutdinov, 2006: Reducing the dimensionality of data with neural network. Science, 313, 504-507, https:// doi.org/10.1126/science.1127647.

-, O. Simon, and Y. W. Teh, 2006: A fast learning algorithm for deep belief nets. Neural Comput., 18, 1527-1554, https:// doi.org/10.1162/neco.2006.18.7.1527.

Huang, W., G. Song, H. Hong, and K. Q. Xie, 2014: Deep architecture for traffic flow prediction: Deep belief networks with multitask learning. IEEE Trans. Intell. Transp. Syst., 15, 2191 2201, https://doi.org/10.1109/TITS.2014.2311123.

Kennedy, J., and R. Eberhart, 1995: Particle swarm optimization. Proc. Int. Conf. on Neural Networks, Perth, WA, Australia, IEEE, 1942-1948, https://doi.org/10.1109/icnn.1995.488968.

Kim, D. H., and W. S. Park, 2005: Neural network for design and reliability analysis of rubble mound breakwaters. Ocean Eng. 32, 1332-1349, https://doi.org/10.1016/j.oceaneng.2004.11.008.

Koesdwiady, A., R. Soua, and F. Karray, 2016: Improving traffic flow prediction with weather information in connected cars: A deep learning approach. IEEE Trans. Veh. Technol., 65, 9508-9517, https://doi.org/10.1109/TVT.2016.2585575.

Längkvist, M., L. Karlsson, and A. Loutfi, 2014: A review of unsupervised feature learning and deep learning for time-series modeling. Pattern Recognit. Lett., 42, 11-24, https://doi.org/ 10.1016/j.patrec.2014.01.008.

Londhe, S. N., and V. Panchang, 2006: One-day wave forecasts based on artificial neural networks. J. Atmos. Oceanic Technol., 23, 1593-1603, https://doi.org/10.1175/JTECH1932.1.

_ S. Shah, P. R. Dixit, T. M. Balakrishnan Nair, P. Sirisha, and R. Jain, 2016: A coupled numerical and artificial neural network model for improving location specific wave forecast. Appl. Ocean Res., 59, 483-491, https://doi.org/10.1016/ j.apor.2016.07.004.

Mahjoobi, J., and E. A. Mosabbe, 2009: Prediction of significant wave height using regressive support vector machines. Ocean Eng., 36, 339-347, https://doi.org/10.1016/ j.oceaneng.2009.01.001.

Makarynskyy, O., 2004: Improving wave predictions with artificial neural networks. Ocean Eng., 31, 709-724, https://doi.org/ 10.1016/j.oceaneng.2003.05.003.

Malekmohamadi, I., R. Ghiassia, and M. J. Yazdanpanah, 2008: Wave hindcasting by coupling numerical model and artificial neural networks. Ocean Eng., 35, 417-425, https://doi.org/ 10.1016/j.oceaneng.2007.09.003.

Mandal, S., and N. Prabaharan, 2006: Ocean wave forecasting using recurrent neural networks. Ocean Eng., 33, 1401-1410, https:// doi.org/10.1016/j.oceaneng.2005.08.007.

Mocanu, E., P. H. Nguyen, W. L. Kling, and M. Gibescu, 2016 Unsupervised energy prediction in a Smart Grid context using reinforcement cross-building transfer learning. Energy Build., 116, 646-655, https://doi.org/10.1016/j.enbuild.2016.01.030.

Özger, M., 2010: Significant wave height forecasting using wavelet fuzzy logic approach. Ocean Eng., 37, 1443-1451, https:// doi.org/10.1016/j.oceaneng.2010.07.009. 
and Z. Sen, 2007: Prediction of wave parameters by using fuzzy logic approach. Ocean Eng., 34, 460-469, https://doi.org/ 10.1016/j.oceaneng.2006.03.003.

Qiu, X. H., Y. Ren, P. N. Suganthan, and G. A. J. Amaratunga, 2017: Empirical mode decomposition based ensemble deep learning for load demand time series forecasting. Appl. Soft Comput., 54, 246-255, https://doi.org/10.1016/j.asoc.2017.01.015.

Ris, R. C., L. H. Holthuijsen, and N. Booij, 1999: A thirdgeneration wave model for coastal regions: 2. Verification. J. Geophys. Res., 104, 7667-7681, https://doi.org/10.1029/ 1998JC900123.

Salakhutdinov, R., 2009: Learning deep generative models. Ph.D. thesis, Dept. of Computer Science, University of Toronto, $84 \mathrm{pp}$.

Shen, F. R., J. Chao, and J. X. Zhao, 2015: Forecasting exchange rate using deep belief networks and conjugate gradient method. Neurocomputing, 167, 243-253, https://doi.org/ 10.1016/j.neucom.2015.04.071.
Taylor, G. W., G. E. Hinton, and S. Roweis, 2006: Modeling human motion using binary latent variables. Advances in Neural Information Processing Systems 19, B. Schölkopf, J. C. Platt, and T. Hoffman, Eds., MIT Press, 1345-1352, https:// papers.nips.cc/paper/3078-modeling-human-motion-usingbinary-latent-variables.pdf.

Tolman, H. L., 1989: The numerical model WAVEWATCH: A third generation model for the hindcasting of wind waves on tides in shelf seas. Communications on Hydraulic and Geotechnical Engineering Rep. 89-2, Delft University of Technology, $87 \mathrm{pp}$.

Tsai, C. P., C. Lin, and J. N. Shen, 2002: Neural network for wave forecasting among multi-stations. Ocean Eng., 29, 1683-1695, https://doi.org/10.1016/S0029-8018(01)00112-3.

WAMDI Group, 1988: The WAM model-A third generation ocean wave prediction model. J. Phys. Oceanogr., 18, 1775-1810, https://doi.org/10.1175/1520-0485(1988)018<1775: TWMTGO $>2.0 . \mathrm{CO} ; 2$. 